\title{
1 Long-range single-molecule mapping of chromatin modification in eukaryotes
}

2 Zhe Weng ${ }^{2,{ }^{*}, \text { Fengying Ruan }}{ }^{2, *}$, Weitian Chen ${ }^{1,2,{ }^{*}, \text { Zhe Xie }}{ }^{2,3}$, Yeming Xie ${ }^{2}$, Chen Zhang ${ }^{2}$,

3 Zhichao Chen ${ }^{1,2}$, Juan Wang ${ }^{2}$, Yuxin Sun ${ }^{2}$, Yitong Fang ${ }^{2}$, Mei Guo ${ }^{2}$, Yiqin Tong ${ }^{2}$, Yaning

$4 \mathrm{Li}^{2}$, Chong Tang ${ }^{2,4,5}$

5

1. College of Life Sciences, University of Chinese Academy of Sciences, Beijing 100049, China

8 2. BGI Genomics, BGI-Shenzhen, Shenzhen 518083, China

9 3. Department of Biology, Cell Biology and Physiology, University of Copenhagen 13,

102100 Copenhagen, Denmark

11 4. Nantong University, Nantong, China, 226000

12 5. Nephrosis Precision Medicine Innovation Center, University of Beihua School of

13 Medicine, Jilin 132011, China

14 "These authors contributed equally to this work.

Keywords: histone, H3K27me3, CTCF, methylation, $\mathrm{m}^{6} \mathrm{~A}$, methyltransferase

Running title: long-range profiling of $\mathrm{H} 3 \mathrm{~K} 27 \mathrm{me} 3$ reveal the heterogenous histone modification

21 Correspondence:

22 Chong Tang

23 Director of technology, BGI Shenzhen, China

24 Phone: 8618025420976

25 Email: tangchong@bgi.com 


\section{Abstract}

2 The epigenetic modifications of histones are essential markers related to the

3 development and pathogenesis of diseases, including human cancers. Mapping histone

4 modification has emerged as the widely used tool for studying epigenetic regulation.

$5 \quad$ However, existing approaches are limited by fragmentation and short-read sequencing

6 represent the average chromatin status in samples and cannot provide information

7 about the long-range chromatin states. We leveraged the advantage of long read

8 sequencing to develop a method "BIND\&MODIFY" for profiling the histone modification

9 of individual DNA fibers. Our approach is based on the recombinant fused protein A-

10 M.EcoGII, which tethers the methyltransferase M.EcoGIl to the protein binding sites and

11 locally labels the neighboring DNA regions through artificial methylations. We

12 demonstrated that the aggregated BIND\&MODIFY signal matches the bulk-level ChIP-

13 seq and CUT\&TAG, verify the single-molecule heterogenous histone modification

14 status, and quantify the correlation between distal elements. This method could be an

15 essential tool in future third-generation sequencing ages. 


\section{Introduction}

2 The genome in each cell of a multi-cellular organism is identical and stays reasonably

3 static, whereas the epigenome is very dynamic ${ }^{1}$. The epigenome varies in different

4 cell types and plays significant roles in various biological processes, such as stem cell

5 differentiation ${ }^{2}$, the nervous system ${ }^{3}$, cell aging and disease ${ }^{4}$. However, current

6 methods developed to study the epigenome are vague and may be less

7 comprehensive than those to study the genome, which can be directly sequenced.

8 The solutions proposed have been based on extracting the epigenome signals by

9 enzymatic or chemical means and isolating either the accessible or protected location.

10 Therefore, much interest has been focused on collecting and comparing genome-wide

11 chromatin accessibility and chromatin modification to locate the specific epigenetic

12 changes that accompany cell differentiation, environmental signaling, and disease

13 development ${ }^{5}$. Next-generation sequencing using DNase I (DNase-seq) and MNase

14 (MNase-seq) treatment has provided the first demonstration that active chromatin

15 coincides with nuclease hypersensitivity ${ }^{67}$. A comparable method is ATAC-seq which

16 screens for the transposon hyperactivity on the accessible chromatin ${ }^{8,9}$. The most

17 recent and advanced theory that proposed to offer a single molecular long-read

18 approach for evaluating chromatin accessibility is a third-generation sequencing

19 technique, referred to as nanopore sequencing, that includes SMACseq ${ }^{10}$, NOME-seq

$20{ }^{11}$, and nanoNOME ${ }^{12}$. Chromatin accessibility assays may address certain epigenetic

21 problems, but the more specific question "which protein regulates chromatin

22 accessibility" cannot be answered. The dynamic chromatin structure is tightly

23 regulated by post-translational histone modifications and binding transcription factors 
1 13. To answer the question: "is a transcription factor bound to a piece of DNA or locate

2 the histone modification" scientists use "ChIP-seq" to find out if a "protein of interest"

3 is bound to a piece of DNA ${ }^{14}$. Large-scale chromatin immunoprecipitation assay

4 (ChIP-seq) utilize specific antibody to precipitate DNA fragments crosslinked with

5 target proteins, followed by direct ultra-high-throughput DNA sequencing ${ }^{15}$. ChIP-seq

6 has facilitated the finding of protein-binding motifs and has allowed us to identify

7 noncanonical protein-binding motifs further. In the literature, these have been

8 extensively investigated to study the biological functions of the histone acetylation and

9 methylations, transcription factors, etc. ${ }^{16-22}$. Currently, the major challenge restricting

10 the use of ChIP-seq is the need for high input DNA amounts. There have been certain

11 methods established to conduct ChIP-seq with low input DNA, for example,

12 ChIPmentation ${ }^{23}$ CUT\&RUN ${ }^{24}$ and CUT\&TAG ${ }^{25}$. For the CUT\&RUN protocol, MNase

13 is fused to protein A (pA-MNase) to guide the chromatin cleavage to antibodies bound

14 to the protein targets at their binding sites across the genome ${ }^{25}$. Conceptually similar

15 work has also been carried out by CUT\&TAG, in which transposons are used instead

16 of the MNase. Many of these techniques and their derivatives have been improved to

17 achieve the single-cell DNA input ${ }^{25-28}$. However, the complex relationship between

18 DNA methylation, chromatin modification, and genome structure variation is often

19 difficult to unravel with a single omics tool. Various methods developed to address this

20 challenge involving applying the bisulfite treatment to the immunoprecipitated DNA

21 fragments during ChIP-seq, which ensures that both DNA methylation information and

22 histone information are procured from the process ${ }^{29-31}$. The above literature review 
1 describes the previous and current attempts to address the status of the protein-bound

2 on DNA.

3 Most ChIP-seq technologies employ the short-read sequencing by next-generation

4 sequencing (NGS), and downstream analysis has been based on the peak calling

5 algorithm with statistical analysis of populated fragments ${ }^{8,9}$. As a result, researchers

6 are then faced with a question - whether the individual chromatin fibers retain the

7 same long-range physical organization as they remove linkages between distal

8 segments. Moreover, due to its use of short-read sequencing, the methylation and

9 structure variation information is limited to the immunoprecipitated genome region. In

10 addition, these short-read sequencing technologies do not utilize non-cleavage

11 labeling methods and lack the capability of long-read sequencing to preserve all

12 necessary epigenetic information in individual chromatin.

13 Our new approach BIND\&MODIFY, by fusing methyltransferase to bind and modify

14 the targeted sites within a long DNA molecule. Furthermore, we developed a single-

15 molecule long-read bound protein mapping sequencing method. This single-molecule

16 method directly examines the protein binding regions, DNA methylation, and complex

17 structure within a single chromatin fiber at multikilobase scales. We used

18 BIND\&MODIFY to study histone methylation and co-methylated histone status in

19 cancer cells. Moreover, we enumerate the detail regulation in the transposon region

20 and bivalent regulatory states, while observing distance coordinated changes in

21 cancer cells. BIND\&MODIFY also allows for the footprinting of other specific

22 transcription factors. We expect future applications of BIND\&MODIFY to enable new 
1 insights into the status of the dynamic regulators of the genome in various

2 experimental systems and sequencing platforms.

4 Results

5 The experimental overview of the BIND\&MODIFY method

6 The rationale of the BIND\&MODIFY method is to indirectly label DNA regions bound

7 with protein of interest via an engineered recombinant fusion protein, protein A-

8 M.EcoGII (pA-M.EcoGII), whose methyltransferase activity can be locally controlled.

9 The recently characterized adenosine methyltransferase M.EcoGII was capable of

10 methylating a broad range of genome DNA in a sequence non-specific manner ${ }^{32}$

11 compared with DamID ${ }^{33}$, which could only methylate the rare GATC motif in the genome

12 (Supplemental Figure 1). Our BIND\&MODIFY approach leverages the power of an

13 engineered recombinant protein, pA-M.EcoGII. First, the recombinant methyltransferase

14 was tethered close to the specific antibody-bound protein of interest, and the adenines

15 in the DNA sequences adjacent to the protein of interest were methylated at $\mathrm{m}^{6} \mathrm{~A}$ in $\mathrm{a}$

16 non-specific manner upon activation (Figure $1 \mathrm{~A})$. As $\mathrm{m}^{6} \mathrm{~A}$ modifications are very limited

17 in the native background of the mammalian chromosome ${ }^{34,35}$, the artificial $\mathrm{m}^{6} \mathrm{~A}$

18 modification, indicating the protein binding motif, could be directly detected by the

19 nanopore ${ }^{36,37}$.

20

21 Details of recombinant protein pA-M.EcoGIl design, construction, and purification steps

22 can be found in the methods section. Briefly, recombinant pA-M.EcoGII is designed by

23 cloning two immunoglobin binding domains of staphylococcal protein $\mathrm{A}$ fused $\mathrm{N}$ - 
1 terminally with M.EcoGII (Figure 1B). The amino acid of the linker region between

2 protein A and M.EcoGII is DDDKEF. The recombinant pA-M.EcoGIl was expressed in

3 the E.coli system and had a molecular weight of $61 \mathrm{kDa}$ (Figure 1C). To access the

4 function of purified recombinant $p A-M$.EcoGll, the $\mathrm{m}^{6} \mathrm{~A}$ dependent restriction enzyme

5 Dpnl digestion was used to verify pA-M.EcoGIl's methylation activity on lambda DNAs.

6 We found that pA-M.EcoGIl processed similar $\mathrm{m}^{6} \mathrm{~A}$ methylation specificity compared to

7 commercially available M.EcoGII (NEB) (Figure 1D). To further evaluate the

8 effectiveness of purified recombinant $\mathrm{pA}-\mathrm{M}$.EcoGII, $\mathrm{m}^{6} \mathrm{~A}$ sensitive restriction enzymes

9 (EcoRV, Pcil, Pvull) were employed to cleave pTXB1 plasmid DNAs treated with pA-

M.EcoGII and commercial M.EcoGII (NEB). Both pA-M.EcoGII and M.EcoGII

11 successfully introduced the $\mathrm{m}^{6} \mathrm{~A}$ methylations into the plasmid, which inhibited the

12 digestion activity of $\mathrm{m}^{6} \mathrm{~A}$ sensitive restriction enzymes (Figure 1E). We performed an

13 ELISA assay to evaluate the potential of pA-M.EcoGll's function of IgG domain binding

14 and observed that it processed similar activity to commercially available protein A

15 (Figure 1F). Taken together, our purified recombinant pA-M.EcoGII has comparable enzymatic activity to commercially available protein A and M.EcoGII.

18 Based on the recombinant pA-M.EcoGll protein, we developed the BIND\&MODIFY protocol (Figure 1G). Briefly, the cells are 1) lightly fixed and tethered to Concanavalin A

20 (ConA)-coated magnetic beads, 2) incubated with primary antibodies, 3) incubated with

21 pA-M.EcoGll with minimal washes, 4) S-adenosylmethionine is added and incubated at

$2237^{\circ} \mathrm{C}$ for $30 \mathrm{~min}$ to initialize the methylation reaction, and then quenched by $0.1 \% \mathrm{SDS}$,

23 5) extraction of DNA by phenol-chloroform and prepare the library for Oxford Nanopore 
1 Technology (ONT) sequencing. After sequencing, the data is processed through

2 genome alignment and $\mathrm{m}^{6} \mathrm{~A}$ signal detection. The $\mathrm{m}^{6} \mathrm{~A}$ possibility cut-off was

3 determined based on background noise (Supplemental Figure 2). The sensitivity and

4 specificity of the $\mathrm{m}^{6} \mathrm{~A}$ signal are around 0.92 and 0.99 , respectively.

6 The validation of BIND\&MODIFY on assigned location in vitro

7 According to previous studies, the frequency of adenosine occurrence on various

8 genomes is once in every $3 b p^{10}$, which was the theoretical resolution of M.EcoGII

9 treated areas. The resolution of BIND\&MODIFY depends not only on the adenosine

10 frequency on the genome but also on the range of the tethered M.EcoGII could reach.

11 Here we present the test that evaluates the signal resolution of BIND\&MODIFY. The

12 first step to developing the method was to synthesize the DNA with an assigned

13 antibody binding site. Then, the PCR was used to introduce the $\mathrm{m}^{5} \mathrm{C}$ modification into

14 the given location on DNAs (Figure 2A). In the following steps, the pA-M.EcoGII

15 recombinant protein was tethered to the designed site with the help of the $\mathrm{m}^{5} \mathrm{C}$

16 antibody. Then the attached M.EcoGII methyltransferase transferred a methyl group to

17 neighboring adenosine after activation. The N6-methyladenosine $\left(\mathrm{m}^{6} \mathrm{~A}\right)$ modified

18 DNAs were sequenced in a Nanopore device, and we performed in-house pipeline

19 data analysis.

20

21 In Figure $2 \mathrm{~B}$, the $\mathrm{m}^{6} \mathrm{~A}$ possibility were plotted along with their genomic location as an

22 indicator of bound pA-M.EcoGII. The regions with high methylation possibility $(>0.53$,

23 Supplemental Figure 2) corresponded to the neighboring areas of the bound pA- 
1 M.EcoGII, where the tethered M.EcoGII could methylate the genome sites within a

2 close distance (Figure 2B). The resolution for detecting pA-M.EcoGIl binding sites was

3 around 100bp (Figure 2B), comparable to the conventional ChIP-seq peak size

4 distribution of 100-300bp (Supplemental Figure 3).

6 The secondary objective of the present study was to investigate the strand specificity

7 of the pA-M.EcoGII modifications. We plotted the methylation possibility, as the

8 indicator of the pA-M.EcoGIl location, on both positive and negative strands (Figure

$92 \mathrm{C})$. We observed that the pA-M.EcoGll binding signal was located exclusively on the

10 negative strand rather than the positive strand. This suggested that the tethered

11 M.EcoGII was in close proximity to the negative strand of DNA and thus preferentially

12 modified that strand. These features make the BIND\&MODIFY method optimal for

13 long-range histone modification studies on the single molecular level and histone

14 modification in a strand-specific manner.

16 BIND\&MODIFY reveals the comparable strand-specific view of the epigenomic

17 regulator on genomic DNA

18 ChIP-seq, CUT\&RUN, and CUT\&TAG detect protein-DNA binding events and

19 chemical modifications of histone proteins ${ }^{38-40}$. Several histone trimethylation states,

20 such as $\mathrm{H} 3 \mathrm{~K} 4 \mathrm{me}$, are well-studied and have been linked to active gene transcription,

21 whereas H3K27me3's relationship with transcription status is more ambiguous ${ }^{41,42 .}$

22 Therefore, we chose to explore the transcription status of H3K27me3 status in the

23 breast cancer cell line MCF-7, using ChIP-seq and BIND\&MODIFY. The average read 
1 length of Nanopore was around 2kb (Supplemental Figure 4), and the correlation of

2 the experimental replicates was 0.90 (Supplemental Figure 5).

4 To validate the H3K27me3 signal as determined by BIND\&MODIFY, we first evaluated

5 the consistency between that obtained from BIND\&MODIFY and the conventional

6 methods. The signals ascertained by both the conventional method (ChIP-seq) and

7 the proposed BIND\&MODIFY method provided the similar H3K27me3 position signals

8 in various genome scales (Figure 3A, Supplemental Figure 6). Over 95\% of the high

9 confidence position signals gathered using ChIP-seq were also observed using

10 BIND\&MODIFY (Supplemental Figure 7). By analyzing the regional signal strength, we

11 found that the BIND\&MODIFY signal strength for $\mathrm{m}^{6} \mathrm{~A}$ counts was strongly correlated

12 with ChIP-seq peak signal intensity (Figure 3B). Taken together, these

13 BIND\&MODIFY results exhibited a range of values comparable with conventional

14 ChIP-seq.

16 Some studies have observed an inverse relationship between H3K27me3 density in

17 transcription start sites and gene expression ${ }^{41,42}$. To profile the enrichment of

18 H3K27me3 within genomic regions, enrichment data were visualized using ChIP-seq

19 H3K27me3 peak centered signal plots. Overall, the BIND\&MODIFY results are

20 consistent with those of ChIP-seq (Figure 3C). In the TSSs of low expression genes

21 (bottom expression quantile), H3K27me3 was prominently depleted around the TSS

22 with a distinctive enrichment directly downstream and upstream of the TSSs (Figure

23 3D). The bimodal pattern is relatively consistent in both ChIP-seq and BIND\&MODIFY 
1 results. Good agreement was also found when comparing results from this work

2 against published data 4344 . Further analysis was performed to determine whether the

3 histone trimethylation exhibited strand specificity using the BIND\&MODIFY method.

4 Subsequently, we found that H3K27me3 was prominently enriched downstream of

5 TSS in the positive strand and upstream of TSS in the negative strand, contributing to

6 form the bimodal pattern around TSSs (Figure 3E). An example of the gene is

7 illustrated in Supplemental Figure 8. In contrast, we did not observe the significant

8 strand-specific pattern around the TSSs of high expression genes (Supplemental

9 Figure 8).

10 As a critical regulator of genome organization, the CCCTC-binding factor (CTCF) has

11 been characterized as a DNA-binding protein with essential functions in maintaining

12 the topological structure of chromatin and inducing gene expression ${ }^{45}$ (Supplemental

13 Figure 9A). In CUT\&TAG data, we identified the strong CTCF binding sites 100bp

14 upstream of the TSSs of actively expressed genes (Supplemental Figure 9B). In

15 contrast, the CTCF binding signal around the TSSs of inactive genes was distributed

16 evenly (Supplemental Figure 9C). Compared with CUT\&TAG, BIND\&MODIFY

17 presented a similar CTCF signal pattern around TSS in active and inactive genes.

18 Further analysis was done to see whether the antibody-bound CTCF domains

19 maintained selective proximity to either one strand (Supplemental Figure 9D). We

20 found that the CTCF signal on the negative strand was sharper and more potent than

21 on the positive strand and even more consistent with the CUT\&TAG signal pattern

22 (Supplemental Figure 9E), suggesting the antibody-bound domain was in close

23 proximity to the negative strand. Based on protein crystal structure analysis ${ }^{46}$, the 
1 antibody-bound domain is the C terminal of the CTCF and spatially closer to the

2 negative strand, further supporting this hypothesis (Supplemental Figure 10).

3 These results have demonstrated that the BIND\&MODIFY method has exhibited that it

4 is capable of mapping the histone modifications in a manner comparable with

5 conventional methods, in addition to highlighting the challenges associated with a

6 strand-specific view of epigenomic regulators on genome DNA

9 Long-range sequencing resolves the retrotransposon regions with high

10 resolution

11 The retrotransposon composed of repeated sequences can be integrated elsewhere in

12 a genome and has performs various critical biological functions. The three major

13 retrotransposon orders are long terminal repeat (LTR) retrotransposons, long

14 interspersed elements (LINEs), and short interspersed elements (SINEs). Due to the

15 repeated sequence of retrotransposon, the short-read sequencing data exhibits

$1640 \sim 60 \%$ multiple mapping rate in these complex regions (Supplemental Figure 11).

17 The multiple mapping in retrotransposons would cause signal noise or signal loss in

18 the ChIP-seq (Figure 4A). Compared with ChIP-seq, the BIND\&MODIFY significantly

19 improves the unique mapping rate (Supplemental Figure 11), by taking advantage of

20 long-range sequencing, which generates reads that may span the entire length of full

21 transposon and avoid multiple mapping issues. Considering these advantages, the

22 long-range sequencing was also used to identify the novel transposon deletion and

23 insertions ${ }^{47}$. There have been minimal studies regarding the H3K27me3 on 
1 retrotransposons ${ }^{48}$. We visualized the H3K27me3 status on LTR, SINE, and LINE

2 using retrotransposon-centered plots, including an upstream/downstream 300bp of

3 similar size retrotransposons (Supplemental Figure 12). In the ChIP-seq, we observed

4 the very high background noise lacking any peak signal on LTR regions (Figure 4B).

5 In contrast, the BIND\&MODIFY displayed the H3K27me3 binding signal on the LTR

6 region, providing a 10X better signal resolution compared to ChIP-seq (Figure 4B).

7 While analyzing another type of retrotransposon, SINE, ChIP-Seq lost the H3K27me3

8 signal in SINEs. However, BIND\&MODIFY displayed two clear peak signals in the

9 SINEs (Figure 4C-D). In addition, these results are consistent well with existing

10 studies regarding H3K27me3 activity on SINEs ${ }^{48}$.

11 The short interspersed nuclear element (SINE) B1 has insulator activity mediated by

12 the binding of specific transcription factors along with the insulator-associated protein

13 CTCF ${ }^{49}$. A genome-wide analysis of CTCF binding sites in the human and mouse

14 genomes discovered that many CTCF binding sites are derived from TE sequences ${ }^{50}$.

15 Concordant with previous reports, the BIND\&MODIFY exhibited a clear CTCF signal in

16 the SINEs, which was absent in the ChIP-Seq data (Supplemental Figure 13). In

17 summary, BIND\&MODIFY is capable of taking advantage of the long-range

18 sequencing to precisely map histone modifications and protein-DNA interactions in the

19 challenging complex genome regions.

21 The histone modification status on single-molecular resolution

22 The conventional CUT\&TAG and ChIP-seq methods are based on statically

23 calling the peak of the enriched read in a specific region ${ }^{51}$. Recent single- 
1 molecule and single-cell measurements of histone accessibility suggest that

2 using ATAC-seq to evaluate cell populations represent an ensemble average of

3 distinct nucleosome states ${ }^{52}$. An essential attribute of the BIND\&MODIFY

4 technique, is that it measures the histone modification in single-molecular

5 resolution by taking advantage of the slight variance (Supplemental Figure 14),

6 thereby increasing the cumulative possibility of segments (Supplemental Figure

7 15).

8 We then hypothesized whether BIND\&MODIFY could reveal the different

9 H3K27me3 statuses by investigating the chr20:52223000-52225500 loci. The

10 chr20:52223000-52225500 loci have modulated the IncRNA LOC105372672

11 and Zinc finger protein 217 (ZNF217) and has been demonstrated as a

12 prognostic biomarker and therapeutic target during breast cancer progression

1353,54 . The conventional ChIP-Seq enriched the H3K27me3 bound motif by

14 antibody-guided amplification, which could be overrepresented (Figure 5A).

15 The baseline signal of this region was 6 , which was considered to be the

16 background noise in the experiments (Figure 5A). In contrast, the

17 BIND\&MODIFY presents a strikingly comprehensive picture of the H3K27me3

18 in this area. Remarkably, super-resolution of BIND\&MODIFY uncovered

19 remarkable three different epigenetic states (Figure 5B): a repressed state in

20 which most histones were methylated, inhibiting the gene expression contained

21 within the genome region; a poised state in which half of the histones were

22 methylated, which permit the transition from the inactive state to the active

23 state; and an active state largely devoid of histone trimethylation. Some of the 
1 H3K27me3 could only be observed in the subpopulation of the genome fibers,

2 suggesting the highly heterogenous histone methylation in cancer cells. The

3 signal peaks (chr20: 52225000-52225500) in ChIP-seq sum up the H3K27me3

4 loci in two different states. The subpopulation of inactive fully methylated

5 chromatin leads to the baseline signal in ChIP-seq, which was thought to be

6 the background noise in the experiment. Our findings, which support three

7 epigenetic states, align with the results reported by other publications in

8 literature ${ }^{55-58}$. Many genome regions in cancer cells harbor a distinctive

9 histone modification signature that combines the activating histone H3 Lys 4

10 trimethylation (H3K4me3) mark and the repressive H3K27me3 mark. The

11 poised states with these bivalent domains, which are considered to be linked to

12 poise the expression of developmental genes, permitting timely activation

13 (activate form) while maintaining repression (repressed state) in the absence

14 of differentiation signals ${ }^{55}$. In contrast to the heterogeneous behaviors seen

15 above, the gene desert areas without gene transcription activity processed only

16 one repressed state wherein most histones were trimethylated (Supplemental

17 Figure 16). The previous bivalent model was established by finding the

18 overlapped H3K4me3 and H3K27m3 average peaks in NGS but not

19 acknowledge their poised status. These findings reinforce the general belief

20 that bivalent histone modification regulates gene expression.

21 However, the single molecular statuses of each gene are challenging to be

22 quantified. Therefore, we could not obtain a big-picture view of global genes,

23 summarizing a more comprehensive perspective with specific examples and 
1 details. Consequently, we used the mean methylation ratio of one molecule to

2 present the methylation status of this molecule (Supplemental Figure 17), and

3 the heterogeneity of the genes could be visualized by a series of mean

4 molecular methylations (Figure 5B, gradient line on the right panel). We

5 summarized the H3K27me3 heterogeneity of genes in Chr20 (Figure 5C). 95\%

6 of genes display a homogenous $\mathrm{H} 3 \mathrm{~K} 27 \mathrm{me} 3$ regulation status with minimal

7 heterogeneity among molecules. Only one cluster with 31 genes (Figure 5C,

8 cluster 3 ) adequately demonstrated the very high heterogeneity of H3K27me3

9 regulation, illustrating its active, repressed, and poised state. These genes are

10 under typical bivalent mode regulation of H3K27me3. Using gene ontology

11 analysis, we found that these genes were enriched in the breast cancer-related

12 pathways, for example, the VEGF singling pathway ${ }^{59}$, B cell receptor signaling

13 pathway ${ }^{60}$, PD-L1 checkpoint pathway ${ }^{61}$,etc. (Supplemental Figure 18). The

14 bivalent mode regulation in these pathways might elucidate the mechanisms

15 involved in tumor heterogeneity, evolution, drug resistance, immune evasion,

16 and the cause of metastasis.

18 The BIND\&MODIFY reveals the long-distance correlation of regulators

19 In the transition from a chromatin repressed state to an activated state, the

20 H3K27me3 changed its rhythmics. The region (chr20: 52223500-52224200)

21 was first devoid of H3K27me3 and then adjacent areas concurrently removed

22 any histone trimethylation during the next step, suggesting the possibility of a

23 synergic regulatory switch at this location. To quantify distance 
1 (anti)correlation between chromatin trimethylation states, we developed a

2 modified correlation coefficient (CC) metric for assessing the degree of

3 trimethylation correlation between genomic regions. Average CC profiles

4 centered on statically positioned nucleosomes revealed a detectable

5 correlation between nucleosome positions up to 100bp (Supplemental Figure

6 19). These observations are consistent with the assumption that nucleosomes

7 impose restrictions on one another, resulting in a short-range correlation

8 between nucleosome footprints that dephases over longer ranges ${ }^{10}$.

9 Furthermore, CC analysis of DNA confirmed the long-range positive correlation

10 between the promoter region and this upstream, downstream element (Figure

116 A, Supplemental Figure 20).

12 However, the CC index is only suitable for describing the distance correlation

13 of H3K27me3 for single genes. To further explore the biological significance of

14 distance correlation, we developed the new DE index (Distance Effect index) to

15 quantify the distal effects of each genomic locus (Supplemental Figure 21).

16 The higher DE index means that these target genomic loci have a stronger

17 distal correlation, suggesting that the regulatory mechanisms present on these

18 sites could impact a larger area. Therefore, the genetic region of interest could

19 be presented as a series of DE indices on the corresponding loci. We plotted

20 the DE index of all the transcription regions ( $2 \mathrm{~kb}$ upstream of TSS) on chr20

21 (Figure 6B). There were several DE patterns consistent with H3K27me3 in the

22 transcription region. Some transcription initiation sites had a powerful DE index

23 signal, which suggested the rhythmic change in the more extensive area and 
1 its substantial regulator impact on the distal genomic region (Figure 6B).

2 Consistent with studies that have associated H3K27me3 with transcriptional

3 repression ${ }^{62}$, a strong DE index signal for $\mathrm{H} 3 \mathrm{~K} 27 \mathrm{me} 3$ was related to lower

4 gene expression (Figure 6B). Based on gene ontology analysis, the

5 dysregulation of these genes was associated with cancer pathways, such as

6 those for ubiquitin dysregulation, Apelin signaling, proteasome, Oxytocin

7 signaling, etc (Supplemental Figure 22).

8 We further plotted the distance correlation of transcription regions with CTCF

9 regulators. The the areas where the transcription initiation sites have strong

10 DE index signal (Figure 6C, Cluster 3) indicate that the CTCF on those sites

11 may synergically affect the distal genomic sites. Compared with the gene

12 expression profile, some gene expression is strongly enhanced. By gene

13 ontology analysis, these enhanced distance regulations were also related to

14 the cancer-promoted pathways such as those for EGFR tyrosine kinase

15 inhibitor resistance, GABAergic synapse, Hippo signaling pathway, etc.

16 (Supplemental Figure 23). In addition, the observed enhanced distance

17 regulation may have elucidated the super-enhancer/suppressor effects, which

18 do affect the gene region up to $8 \mathrm{~kb}^{1}$.

19 Overall, the BIND\&MODIFY was able to demonstrate the highly heterogenous

20 status of histone modification in the cancer cells and the long-range interaction

21 of these histone modifications.

22 
1 In literature, ChIP-seq and CUT\&TAG have been essential epigenetic study tools for many years, for example, histone modification, transcription factor binding, and much more. However, these methods suffer from the limitations that the detected signals are represented using common immunoprecipitation enriched DNA fragments in common without considering the heterogeneity in DNA molecules. The short-read sequences

6 also prevent the possibility of studying the long-range interactions in multi-omics

7 epigenome studies. We designed a new method, "BIND\&MODIFY," by using the

8 recombinant $\mathrm{pA}-\mathrm{M}$.EcoGIl methyltransferase to non-fragmented labeling the local DNA

9 regions. Through this method, we were able to simultaneously profile multi epigenomic

10 information on a truly unbiased genome-wide scale, measure the underlying distribution

11 of the histone modifications based on single molecular resolution, and identified loci

12 exhibiting significant correlation.

13 BIND\&MODIFY generated a similar general signal trend for the H3K27me3 loci to the

14 widely used ChIP-seq. Despite the significant commonalities between the results of the

15 two methods, relatively small signal variations were observed in terms of the variable

16 peak signal strength. We postulate that these were likely caused by the different

17 fundamental principles between BIND\&MODIFY and conventional ChIP-seq. The

18 BIND\&MODIFY enables unbiased profiling of the DNA molecule without

19 immunoprecipitation enrichment, thereby reflecting the true nature of the epigenome.

20 Compared with that method, ChIP-seq only amplifies the common loci signals among

21 most DNA molecules by immunoprecipitation enrichment and neglects the loci signals

22 of in individual DNA molecules. 
1 Moreover, the different measuring units to present in signal strength also led to the

2 difference. One drawback of the BIND\&MODIFY is that it sequences the non-signal

3 regions, which is unavoidable, thereby requiring a higher sequencing depth.

4 Fortunately, the nanopore throughput is increasing rapidly, while selective enrichment

5 methods are also becoming increasingly available. Augmenting the read length are also

6 helpful, especially for analyzing the distance correlation of distal regulatory elements.

7 We also compared our method with CUT\&TAG. The significant difference is that the

8 transposon utilized in CUT\&TAG is not reusable once inserted into the DNAs. In

9 contrast, the methyltransferase used in BIND\&MODIFY could methylate the multiple

10 regional adenosines, enhancing the signal strength. CUT\&TAG also requires a

11 secondary antibody to tether more transposons to the location and increase the chance

12 of DNA insertion. In BIND\&MODIFY method, we found that the secondary antibody is not necessary. Others may suggest that the reusable methyltransferase may increase

14 the false-positive signals in non-target regions. However, during in vitro evaluation, we

15 found the only scattered signals in the non-targeted area, but no clustered signals, which were consistent with identifiable loci signal (Figure 2). In addition, during in vivo

17 evaluation, the genomic DNA was not movable by fixation, and the DNA

18 methyltransferase can only activate locally (Figure 3). Therefore, any false-positive

19 signal of in vitro and in vivo can be safely neglected based on these studies.

20 Base-calling is another area of future improvement. We also used the Pacbio

21 sequencing data (specificity 0.99 ) to train our $\mathrm{m}^{6} \mathrm{~A}$ calling algorithm with nanopore

22 sequencing. Both sensitivity and specificity with regard to nanopore $\mathrm{m}^{6} \mathrm{~A}$ detection were 
1 satisfactory. We are also trying to call the native $\mathrm{m}^{5} \mathrm{C}$ and artificially labeled $\mathrm{m}^{6} \mathrm{~A}$

2 simultaneously and analyze the relationship between histone modification and DNA

3 methylation. To our surprise, the artificially labeled $\mathrm{m}^{6} \mathrm{~A}$ did not significantly affect the

$4 \quad \mathrm{~m}^{5} \mathrm{C}$ detection efficiency significantly, and the $\mathrm{m}^{5} \mathrm{C}$ correlation using bisulfite sequencing

5 was 0.8 (Supplemental Figure 24). The relationship between $5 \mathrm{mC}$ and histone

6 modification in single molecules should be determined in much deeper sequencing

7 depth. Alternatively, we could also use the Pacbio, which reads one nucleotide at a time

8 without the neighboring nucleotide signal interference. Overall, the simultaneous

9 detection of $5 \mathrm{mC}$ and its regulator position in the single-molecule level is applicable with

10 BIND\&MODIFY.

11 Possible endogenous methylation in mammalian genomes also represents the potential

12 confounding factor in our analysis. By examining the data of IgG control genomic DNA, we found that the quantity of $\mathrm{m}^{6} \mathrm{~A}$ in original DNA is thousands of times fewer than in

14 the treated samples, suggesting the minimal effect of the endogenous methylation. To

15 determine the thresholds for the possibility of real $m^{6} A$ and background noise, the $\lg G$

16 control sample data was used to determine and filter out the background noise signal

17 distribution is below 0.53 , which could significantly improve the $\mathrm{m}^{6} \mathrm{~A}$ calling specificity 18 and sensitivity.

19 However, there are also some species where $\mathrm{m}^{6} \mathrm{~A}$ occurs endogenously and strongly 20 correlated with the binding motif of the histone modification. Modifications targets such

21 as $\mathrm{m}^{5} \mathrm{C}, \mathrm{m}^{4} \mathrm{C}$, cytidine deamination, or 5-glucomethyaltion are among the potential

22 future alternatives in such cases. Also, some artificial SAM could be used to introduce 
1 the biotin to the specific sites may strongly avoid the endogenously confounding signals.

2 Finally, we believe that the integration of BIND\&MODIFY into a single-molecule multi-

3 omics assay represents a fruitful direction that will benefit future research. With the

4 method, we are able to simultaneously label the two or more proteins located in the

5 single DNA molecule with the different modification labels. Then the protein interaction

6 distance on the genome would be measurable in this method, providing a potential

7 breakthrough in the protein-protein interaction in single-molecule genome scales. In

8 principle, similar approaches may also apply to the individual RNA molecules to study

9 the RNA binding proteins. We expect this technology to contribute to the essential new

10 class of tools that will improve the simultaneous study of multi-epigenomics.

\section{Methods}

13 Cell culture and antibodies

14 Human mammary gland carcinoma cell line MCF-7 were obtained from ATCC. MCF-7

15 were grown in DMEM (Gibco,11995065) supplemented with 10\% FBS (Gibco,10099141),

$16 \quad 0.01 \mathrm{mg} / \mathrm{ml}$ insulin (HY-P1156, MedChemExpress), and 1\% penicillin-streptomysin (Gibco,

17 15140122). Cell line was regularly checked for mycoplasma infection (Yeasen,

18 40612ES25). We used the following antibodies: H3K27me3 (Cell Signaling Technology,

19 9733), Guinea Pig anti-Rabbit IgG (Heavy \& Light Chain) antibody, Anti-CTCF Antibody

20 (sigma,07-729-25UL), Protein A Antibody, pAb, Chicken (A00729-40, GenScript).

21

22 Recombinant protein preparation of protein A-M.EcoGII 
1 pTXB1 vector (NEB, N6707S) was used as the protein expression backbone.

2 Downstream of the lac operator, a ribosome binding site and three FLAG epitope tags

3 were introduced, followed by two lgG-binding domains of staphylococcal protein $\mathrm{A}$

4 encoding sequence, which was synthesized based on the previous work (Addgene,

5 124601). The M.EcoGll encoding sequence (Addgene, 122082) was also synthesized

6 based on its original discovery. The amino acid linker sequence between the C-terminus

7 of protein $A$ and $\mathrm{N}$-terminus of M.EcoGIl is DDDKEF. The sequenced plasmid was

8 transformed into C3013 competent cells (NEB) following the manufacturer's protocol.

9 Each colony tested was inoculated into a $1 \mathrm{~mL}$ LB medium, and growth was continued at

$1037{ }^{\circ} \mathrm{C}$ for $2 \mathrm{~h}$. That culture was used to start a $50 \mathrm{~mL}$ culture in $100 \mu \mathrm{g} / \mathrm{mL}$ carbenicillin

11 containing LB medium and incubated on a shaker until the cell density reached an A600

12 of 0.6 , whereupon it was chilled on ice for $30 \mathrm{~min}$. Fresh IPTG was added to $0.25 \mathrm{mM}$ to

13 induce expression. Then the culture was incubated at $27^{\circ} \mathrm{C}$ on a shaker for $16 \mathrm{~h}$. The

14 culture was then collected by centrifugation at $10,000 \mathrm{rpm}, 4{ }^{\circ} \mathrm{C}$ for $30 \mathrm{~min}$, the

15 supernatant was discarded. The bacterial pellet was frozen in a dry ice-ethanol bath and

16 stored at $-70{ }^{\circ} \mathrm{C}$. The frozen pellet was resuspended in $20 \mathrm{~mL}$ chilled HEGX Buffer (20

$17 \mathrm{mM}$ HEPES-KOH at pH 7.2, $0.8 \mathrm{M} \mathrm{NaCl}, 1 \mathrm{mM}$ EDTA, 10\% glycerol, 0.2\% Triton X-100)

18 including $1 \times$ Roche Complete EDTA-free protease inhibitor tablets and lysed using a high-

19 pressure cell disrupter (JNBIO, China). Cell debris was removed by centrifugation at

$2010000 \mathrm{rpm}$ for $30 \mathrm{~min}$ at $4^{\circ} \mathrm{C}$, and the supernatant was loaded onto a column equipped

21 with chitin slurry resin (NEB, S6651S), then incubated the column on a rotator at $4{ }^{\circ} \mathrm{C}$

22 overnight. The unbound soluble fraction was drained, and the columns were rinsed with

2320 mL HEGX containing Roche Complete EDTA-free protease inhibitor tablets. The chitin 
1 slurry was transferred to a $15 \mathrm{~mL}$ conical tube and resuspended in Elution buffer (10 $\mathrm{mL}$

2 HEGX with $100 \mathrm{mM}$ DTT). The tube was placed on the rotator at $4{ }^{\circ} \mathrm{C}$ for $\sim 48 \mathrm{~h}$. The

3 eluate was collected and concentrated using an Amicon Ultra-4 Centrifugal Filter Units

$410 \mathrm{~K}$ (Millipore, UFC801096), and sterile glycerol was added to make a final 50\% glycerol

5 stock of the purified protein. The fusion protein has stored the protein at $-80^{\circ} \mathrm{C}$.

6

7 Size characterization of recombinant protein pA-M.EcoGII

8 The size of purified pA-M.EcoGIl recombinant protein was characterized by C Coomassie

9 blue staining by resolving the protein in 7.5\% SDS-PAGE gel.

11 ELISA of recombinant protein pA-M.EcoGII

12 To verify the binding efficiency of pA-M.EcoGll, we performed an ELISA assay in vitro.

13 The purified recombinant protein or commercial M.EcoGII (NEB, M0603S) was diluted

14 with coating buffer (0.05 $\mathrm{M} \mathrm{NaHCO}_{3}$ buffer, $\mathrm{pH}$ 9.2). The 96 well high binding plate

15 (Greiner Bio-one, 655061) was coated with $100 \mu \mathrm{L}$ pA-M.EcoGIl or commercial M.EcoGII

(NEB,M0603S) $(1: 120,1: 480$ of stock $10 \mathrm{mg} / \mathrm{ml})$, negative control, commercial ProteinA

17 (ThermoFisher, 21181) $(1: 120,1: 480$ of stock $10 \mathrm{mg} / \mathrm{ml})$ in coating buffer per well for $4 \mathrm{~h}$

18 at room temperature. Further, $200 \mu \mathrm{L}$ SuperBlock $^{\mathrm{TM}}$ (TBS.) Blocking Buffer - Blotting

19 (Invitrogen,37537) was added to each well and incubated for $2 \mathrm{~h}$ at room temperature.

20 Then each well was washed $5 \mathrm{X}$ with $380 \mu \mathrm{L}$ washing buffer $(0.14 \mathrm{M} \mathrm{NaCl} ; 0.01 \mathrm{M}$

$21 \mathrm{PO}_{4} ; 0.05 \%$ Tween 20; pH 7.4). After that $95 \mu \mathrm{L}$ Secondary Antibody (1:10000 in PBSB)

22 was added to each well and incubated for $1 \mathrm{~h}$ at room temperature. The plate was washed

$234 \mathrm{X}$ with $380 \mu \mathrm{L}$ washing buffer (0.14 M NaCl; $0.01 \mathrm{M} \mathrm{PO}_{4} ; 0.05 \%$ Tween 20; pH 7.4). 90 
$1 \mu \mathrm{L}$ TMB substrate solution was added and incubated for $15 \mathrm{~min}$ at room temperature in

2 the dark. Finally, $90 \mu \mathrm{L}$ stop buffer $\left(1.8 \mathrm{~N} \mathrm{H}_{2} \mathrm{SO}_{4}\right)$ was added to stop the color development

3 and read immediately at 450nm (yellow color) using FLUOstar® Omega Plate Reader by

4 BMG LABTECH.

6 In vitro methtransferase activity of pA-M.EcoGII recombinant protein

7 To access the in vitro methylation efficiency of pA-M.EcoGII recombinant protein, $m^{6} A$

8 methylation-sensitive restriction enzyme Dpnl was used to probe the adenine methylation

9 at GATC motif of $7 \mathrm{~kb}$ unmethylated dsDNA. The $7 \mathrm{~kb}$ dsDNA substrate was PCR

10 amplified from lambda DNA. For the methyltransferase reactions, each $50 \mu l$ reaction

11 volume was assembled on ice and contained $1 \mu \mathrm{g} 7 \mathrm{~kb}$ unmethylated dsDNA, $1 \mathrm{X}$ Cutsmart

12 buffer, $640 \mu \mathrm{M}$ SAM, and $4 \mu \mathrm{L}$ of pA-M.EcoGIl recombinant protein or commercial M.

13 EcoGll, then the mixture was incubated at $37^{\circ} \mathrm{C}$ for $1 \mathrm{~h}$. The methylated product was

14 purified using 0.6X Ampure XP (BECKMAN COULTER, A63882). $1 \mu$ l of Dpnl (NEB) was

15 added to the reaction mixture to further incubate at 37 degrees for $10 \mathrm{~min}$. Dpnl cutting

16 efficiency was examined by $1 \%$ agarose gel electrophoresis.

18 To assess the specificity of pA-M.EcoGll recombinant protein methylation and its 19 effectiveness at inhibiting restriction endonucleases, we carried out restriction analyses 20 using an unmethylated $7 \mathrm{~kb}$ linear dsDNA template, which was PCR amplified from pTXB1.

21 One enzyme known to be insensitive to dA methylation (BamHI) and three enzymes

22 (EcoRV, Pcil, Pvull) that cleave different base-pair sequences, the activities of which are

23 known to be blocked by adenine methylation. The commercial M.EcoGI and pA- 
1 M.EcoGIl recombinant protein methyltransferase reactions on the $7 \mathrm{~kb}$ linear dsDNA were

2 carried out the same as described above. All restriction endonucleases used in this study

3 were purchased from NEB. For the restriction enzyme digest reaction, each $30 \mu \mathrm{L}$

4 reaction volume contained $500 \mathrm{ng}$ methylated dsDNA, the appropriate digestion buffer,

5 time, and amount of enzyme following the manufacturer's protocol of NEB. The enzymes

6 were inactivated at $80{ }^{\circ} \mathrm{C}$ for 20 minutes. All sample was loaded to $1 \%$ agarose gel for 7 analysis.

9 In vitro pA-Tn5 transposome preparation

10 The pA-Tn5 was purchased from Vazyme (Vazyme, S603). To generate the pA-Tn5

11 adapter transposon, $7 \mu \mathrm{L}$ of a $50 \mu \mathrm{M}$ equimolar mixture of pre-annealed Tn5MEDS-A and

12 Tn5MEDS-B oligonucleotides, $40 \mu \mathrm{L}$ of $7.5 \mu \mathrm{M}$ pA-Tn5 fusion protein, and $28 \mu \mathrm{L}$ coupling

13 buffer were mixed. The mixture was incubated for $1 \mathrm{~h}$ on a Thermocycler at room

14 temperature and then stored at $-20^{\circ} \mathrm{C}$.

CUT\&TAG for bench-top application

17 Gently resuspend and withdraw enough of the slurry such that there will be $10 \mu L$ for each

18 final sample. Place the tube on a magnet stand to clear (30 s to $2 \mathrm{~min}$ ). Withdraw the

19 liquid, and remove it from the magnet stand. Add $1.5 \mathrm{~mL}$ Binding buffer (20 mM HEPES

$20 \mathrm{pH} 7.5,10 \mathrm{mM} \mathrm{KCl}, 1 \mathrm{mM} \mathrm{CaCl}_{2}$, and $1 \mathrm{mM} \mathrm{MnCl}_{2}$ ), mix by inversion or gentle pipetting,

21 remove liquid from the cap and side with a quick pulse on a microcentrifuge. Resuspend

22 in a volume of Binding buffer equal to the volume of bead slurry (10 $\mu \mathrm{L}$ per final sample).

$2310 \mu \mathrm{L}$ of activated beads were added per sample and incubated at room temperature for 
$115 \mathrm{~min}$. Cells were harvested, counted and centrifuged for $3 \mathrm{~min}$ at $600 \times \mathrm{g}$ at room

2 temperature. 500000 cells were washed 2X in $1.5 \mathrm{~mL}$ Wash Buffer (20mM HEPES pH

$37.5,150 \mathrm{mM} \mathrm{NaCl}, 0.5 \mathrm{mM}$ Spermidine, $1 \times$ Protease inhibitor cocktail), after that the cells

4 were resuspended in $1.5 \mathrm{~mL}$ Wash Buffer by gentle pipetting in a $2 \mathrm{~mL}$ tube. The unbound

5 supernatant was removed by placing the tube on the magnet stand to clear and pulling

6 off all of the liquid. The bead-bound cells were resuspended with $50 \mu \mathrm{L}$ Dig-Wash Buffer

7 (20mM HEPES pH 7.5, 150mM NaCl, 0.5mM Spermidine, 1× Protease inhibitor cocktail,

$8 \quad 0.05 \%$ Digitonin) containing 2mM EDTA and a 1:100 dilution of the appropriate primary

9 antibody. The primary antibody was incubated on a rotating platform overnight at $4{ }^{\circ} \mathrm{C}$.

10 The primary antibody was removed by placing the tube on the magnet stand to clear and

11 pulling off all of the liquid. The secondary antibody was diluted 1:100 in $100 \mu \mathrm{L}$ of Dig-

12 Wash buffer, and cells were incubated at room temperature for $1 \mathrm{~h}$. Cells were washed

13 using the magnet stand twice for $5 \mathrm{~min}$ in $1 \mathrm{~mL}$ Dig-Wash buffer to remove unbound

14 antibodies.0.04 $\mu \mathrm{M}$ of pA-tn5 was prepared in $150 \mu \mathrm{L}$ Dig-Med Buffer (0.05\% Digitonin,

1520 mM HEPES, pH 7.5, 300 mM NaCl, 0.5 mM Spermidine,1× Protease inhibitor cocktail).

16 After removing the liquid on the magnet stand, $150 \mu \mathrm{L}$ was added to the cells with gentle

17 vortexing, which was incubated with pA-Tn5 at room temperature for $1 \mathrm{~h}$. Cells were

18 washed twice for $5 \mathrm{~min}$ in $1 \mathrm{~mL}$ Dig-Med Buffer to remove unbound pA-Tn5 protein. Next,

19 cells were resuspended in $300 \mu \mathrm{L}$ Tagmentation buffer (10 mM MgCl 2 in Dig-Med Buffer)

20 and incubated at $37^{\circ} \mathrm{C}$ for $1 \mathrm{~h}$. To stop tagmentation, $15 \mu \mathrm{L}$ EDTA, $3 \mu \mathrm{L} 10 \%$ SDS, $2.5 \mu \mathrm{L}$

$2120 \mathrm{mg} / \mathrm{ml}$ proteinase $\mathrm{K}$ was added to $300 \mu \mathrm{L}$ of the sample, which was incubated in a

22 water bath overnight at $55^{\circ} \mathrm{C} .320 \mu \mathrm{L} \mathrm{PCl}$ was added to the tube and mixed by full-speed

23 vortexing for 2 s. The upper phase was transferred to a phase-lock tube. $320 \mu \mathrm{L}$ 
1 Chloroform was added to the tube and inverted $\sim 10 x$ to mix. The tube was Centrifuged

2 for 3 min at room temperature at $16,000 \times \mathrm{g}$. The aqueous layer was transferred to a fresh

$31.5 \mathrm{~mL}$ tube containing $750 \mu \mathrm{L} 100 \%$ ethanol and mixed by pipetting. The tube was

4 Chilled on ice and centrifuged for at least $10 \mathrm{~min}$ at $4{ }^{\circ} \mathrm{C} 16,000 \times \mathrm{g}$. The liquid was

5 removed, and $1 \mathrm{~mL} 100 \%$ ethanol was added to the tube, then centrifuged $1 \mathrm{~min}$ at $4{ }^{\circ} \mathrm{C}$

$616,000 \times \mathrm{g}$. The liquid was carefully poured off and air dry. When the tube is dry, $25 \mu \mathrm{L}$

$710 \mathrm{mM}$ Tris-HCl pH 8, $0.1 \mathrm{mM}$ EDTA was added to the tube and vortex on full of dissolving

8 the genomics DNA

BIND\&MODIFY for bench-top application

11500,000 cells were used in each BIND\&MODIFY assay. Cells were harvested, counted,

12 and centrifuged for $3 \mathrm{~min}$ at $600 \times \mathrm{g}$ at room temperature. Cells were first lightly fixed by

13 adding formaldehyde (ThermoFisher, 28906) to a final concentration of $0.1 \%$ in $1.5 \mathrm{ml}$

14 PBS, and incubated at room temperature for 15min. 2.5M Glycine was added to final

15 concentration of $0.125 \mathrm{M}$ to quench the additional formaldehyde. Fixed cells were then washed twice in $1.5 \mathrm{~mL}$ Wash Buffer (20mM HEPES pH 7.5, 150mM NaCl, 0.5mM

17 Spermidine, $1 \times$ Protease inhibitor cocktail) by gentle pipetting. $10 \mu \mathrm{L}$ of activated beads

18 were added per sample and incubated at room temperature for $15 \mathrm{~min}$. The unbound supernatant was removed, bead-bound cells were resuspended in $100 \mu \mathrm{L}$ Dig-Wash

20 Buffer (20 mM HEPES pH 7.5, 150 mM NaCl, 0.5 mM Spermidine, 1× Protease inhibitor

21 cocktail, 0.05\% Digitonin) containing 2 mM EDTA and a 1:100 dilution of the appropriate

22 primary antibody. Primary antibody incubation was performed on a rotating platform

23 overnight at $4{ }^{\circ} \mathrm{C}$. The primary antibody was removed by placing the tube on the magnet 
1 stand to clear and pulling off all of the liquid. No secondary antibody was used for

2 BIND\&MODIFY. Cells were washed twice using the magnet stand for $5 \mathrm{~min}$ in $1 \mathrm{~mL}$ Dig-

3 Wash buffer. $4 \mu \mathrm{L}$ of pA-M.EcoGll was prepared in $150 \mu \mathrm{L}$ Dig-Wash Buffer. After

4 removing the liquid on the magnet stand, $150 \mu \mathrm{L}$ of $\mathrm{pA}-\mathrm{M}$.EcoGIl containing Dig-Wash

5 buffer was added to the cells with gentle vortexing, which was incubated at room

6 temperature for $1 \mathrm{~h}$. Cells were washed $2 \times$ for 5 min in $1 \mathrm{~mL}$ Dig-Wash Buffer and 1x for

75 min in $1 \mathrm{~mL}$ Low salt buffer (20 mM HEPES pH 7.5, $0.5 \mathrm{mM}$ Spermidine, 1× Protease

8 inhibitor cocktail, $0.05 \%$ Digitonin) to remove unbound pA-M.EcoGIl protein. Next, cells

9 were resuspended in $300 \mu \mathrm{L}$ Reaction buffer (20 mM HEPES pH 7.9, 0.5 mM Spermidine,

$101 \times$ Protease inhibitor cocktail, 0.05\% Digitonin, $10 \mathrm{mM} \mathrm{1M} \mathrm{Mgcl} 2,300 \mathrm{mM}$ sucrose) .The

11 reaction was activated by adding $5 \mu \mathrm{L}$ SAM of $32 \mathrm{mM}$ at $37^{\circ} \mathrm{C}$ in a thermmixer. Additional

$125 \mu \mathrm{L}$ SAM of $32 \mathrm{mM}$ was added to the tube at $7.5 \mathrm{~min}$ and $15 \mathrm{~min}$. The reaction was

13 stopped at 30 minutes by placing the tube on the magnet stand to clear and pulling off all

14 of the liquid. The bead-bound cells was resuspended with $300 \mu \mathrm{L}$ Digestion Buffer(20 mM

15 HEPES pH 7.5, 300 mM NaCl, 0.5 mM Spermidine, 1× Protease inhibitor cocktail, 0.05\%

16 Digitonin, $16.7 \mathrm{mM}$ EDTA, 0.1\% SDS, $0.167 \mathrm{mg} / \mathrm{mL}$ proteinase $\mathrm{K}$ ) and incubated in water

17 bath overnight at $55^{\circ} \mathrm{C}$. The method for genomic DNA extraction was the same as

18 CUT\&TAG for bench-top application.

CUT\&TAG library preparation

21 To amplify libraries, $21 \mu \mathrm{L}$ of CUT\&TAG genomic DNA was mixed with $2 \mu \mathrm{L}$ of a universal

22 i5 and a uniquely barcoded i7 primer, using a different barcode for each sample. A volume

23 of $25 \mu \mathrm{L}$ KAPA HIFI ready mix (KAPA, KK2602) was added and mixed. The sample was 
1 placed in a Thermocycler with a heated lid using the following cycling conditions: $72{ }^{\circ} \mathrm{C}$

2 for $5 \mathrm{~min} ; 98^{\circ} \mathrm{C}$ for $30 \mathrm{~s} ; 14$ cycles of $98^{\circ} \mathrm{C}$ for $10 \mathrm{~s}, 63{ }^{\circ} \mathrm{C}$ for $30 \mathrm{~s}$ and $72{ }^{\circ} \mathrm{C}$ for $15 \mathrm{~s}$;

3 final extension at $72{ }^{\circ} \mathrm{C}$ for $1 \mathrm{~min}$ and hold at $8^{\circ} \mathrm{C}$. Post-PCR clean-up was performed by

4 adding 1.3× volume of Ampure XP beads (Beckman Counter), and libraries were

5 incubated with beads for 5 min at RT, washed twice gently in $80 \%$ ethanol, and eluted in

$630 \mu \mathrm{L} 10 \mathrm{mM}$ Tris pH 8.0. the library was analyzed using Agilent 2100 (2100 Bioanalyzer

7 Instrument, G2939BA). Then the library was sequenced in MGI2000 platform with

8 PE100+100+10 sequencing.

BIND\&MODIFY library preparation

11 The BIND\&MODIFY library was prepared following the manufacturer's protocol of SQK-

12 LSK109 (Nanopore, SQK-LSK109). The library was sequenced in the ONT PromethION

13 platform with R9.4.1 flow cell.

15 Basecalling and DNA methylation calling

16 Reads from the ONT data were performed using megalodon (V2.2.9), which used

17 Guppy basecaller to basecalling and Guppy model config

18 res_dna_r941_min_modbases-all-context_v001.cfg has been released into the Rerio

19 repository was used to identify DNA $\mathrm{m}^{6} \mathrm{~A}$ methylation. megalodon_extras was used to

20 get per read modified_bases from the megalodon basecalls and mappings results. In

21 order to futher explore the accurate threshold of methylation probability, a control

22 sample with almost no $\mathrm{m}^{6} \mathrm{~A}$ methylation was used as background noise, and Gaussian 
1 mixture model was used to fit the methylation probability distribution generated by

2 megalodon.

4 Accessibility score

$5 \quad \mathrm{Hg} 19$ genome and the gene elements were processed into 50bp bin sliding 5bp by

6 Bedtools (v2.27.1) ${ }^{63}$. The accessibility score over multi base-pair windows were

7 calculated as methylation ratio $=\mathrm{m}^{6} \mathrm{~A}$ bases in all covered reads under bin/ adenosine

8 bases in all covered reads under bin. And the accessibility score of each single

9 molecule in the bin was also calculated.

\section{ChIP-seq data processing}

12 Demultipexed fastq files were mapped to the hg19 genome using Bowtie2(2.4.1) ${ }^{64}$ with

13 the following settings: bowtie2 --end-to-end --very-sensitive --no-mixed --no-discordant -

14 -phred33 -I 10 -X 700. peaks were called using MACS2 (v.2.1.0) ${ }^{65}$ with the following

15 settings: -g 12000000-f BAMPE.

17 CUT\&TAG data processing

18 Demultipexed fastq files were mapped to the hg19 genome using Bowtie2 with the

19 following settings: bowtie2 --end-to-end --very-sensitive --no-mixed --no-discordant --

20 phred33 - I $10-X 700$. Because of a constant amount of pATn5 is added to CUT\&TAG

21 reactions and brings along a fixed amount of E. coli DNA, we used bowtie2 with the

22 parameters mentioned above to remove E. coli DNA and conduct normalization 
1 according to the CUT\&TAG tutorial ${ }^{25}$. CUT\&TAG peaks were called using SEACR(1.3)

$2 \quad{ }^{66}$ with default parameters.

$4 \quad$ RNA-seq analysis

5 RNA-seq expected counts of the MCF-7 cell lines in all replicates were corrected to be

6 TPM, the mean TPM of all replicates was used as the expression level of each gene for

7 subsequent analysis.

9 Gene single molecular diversity

10 The accessibility of a single molecule in each gene was calculated, and the value in

11 each gene was sorted from small to large by the gene unit. Then the hierarchical

12 clustering was performed for the diversity of single molecule accessibility of each gene.

13 KOBAS3.0 ${ }^{67}$ was used for KEGG and GO analysis for each cluster.

15 Assess replicate reproducibility

16 To study the reproducibility between replicates, the genome is split into $50 \mathrm{bp}$ bins and

17 sliding 5bp, then a Pearson correlation of the log2-transformed values of $\mathrm{m}^{6} \mathrm{~A}$

18 methylation ratio in each bin is calculated between replicate datasets.

20 SV calling

21 We used NGMLR (v0.2.7) ${ }^{68}$ to compare the read of ONT to the human reference

22 genome of hy19 to get the BAM file for comparison. Then we used samtools (v1.2) to 
1 sort the bam files. The sniffles (v1.0.12) ${ }^{68}$ with the parameter --genotype -T 8 -S 8 were

2 then used to call the structural variation on the bam file created in the previous step.

4 Co-accessibility assessment

5 To evaluate co-accessibility patterns along the genome, we applied COA as follows.

6 Each chromosome in the genome was split into windows of size w. For each such

7 window $(i, i+w)$, we identified another window $(j, j+w)$ such that the span $(i, j, w)$ was

8 covered by $\geq N$ reads. For each single spanning molecule $k$, accessibility scores $(A)$ in

9 each bin were then aggregated and binarized as described above. The local co-

10 accessibility matrix between two windows was calculated:

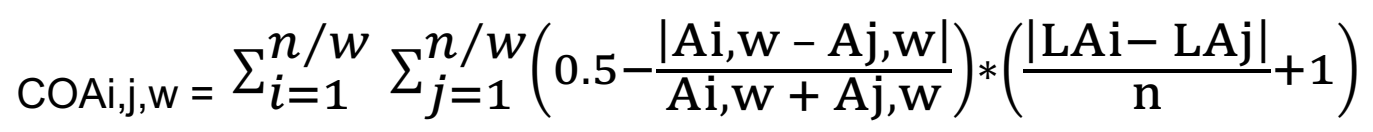

12 where $\mathrm{n}$ is the length of selected region, $\mathrm{L}$ is the location of region.

Data availability

CUT\&TAG for H3K27me3 and CTCF as well as Nanopore raw data are available at China National GeneBank (CNGB) with project number of CNP0001299.

External sequencing datasets.

19 A number of previously published MCF-7 breast cancer datasets were used in this study. ChIP-seq data for CTCF was downloaded from ENCODE with accession

21 ENCSR000DMR, ChIP-seq data for H3K27me3 was downloaded from ENCODE with 
1 accession ENCSR761DLU. The RNA-seq data of MCF-7 was download from the Gene

2 Expression Omnibus (GEO) repository database with the accession number

3 GSE71862.

4

5 Other external datasets.

6 Hg19 genome, short interspersed nuclear elements (SINE) and long interspersed

7 nuclear elements (LINE) region were downloaded from NCBI. TES, TTS and other gene

8 elements were downloaded from the UCSC Table Browser. MCF-7 CTCF binding site

9 was downloaded from the CTCFBSDB_v2.0 ${ }^{69}$.

\section{Acknowledgment}

12 This research was supported by the Science, Technology, and Innovation Commission

13 of Shenzhen Municipality (grant number JSGG20170824152728492). The supporter had

14 no role in designing the study, data collection, analysis, and interpretation, or in writing 15 the manuscript.

\section{Author contributions}

18 CT designed and supervised the experiments. ZW and FR perform the lab experiments;

19 WTC and CT perform the bioinformatics data analysis. All others joined the data analysis.

\section{Competing interest}

22 The authors declare no competing interests. 
bioRxiv preprint doi: https://doi.org/10.1101/2021.07.08.451578; this version posted July 15,2021 . The copyright holder for this preprint (which was not certified by peer review) is the author/funder, who has granted bioRxiv a license to display the preprint in perpetuity. It is made available under aCC-BY-NC-ND 4.0 International license.

1 
1. What Is Epigenomics? I SpringerLink. (2021).
2. Toh, T.B., Lim, J.J. \& Chow, E.K. Epigenetics in cancer stem cells. Mol Cancer 16,
3. Jiang, Y. et al. Epigenetics in the Nervous System. The Journal of Neuroscience 28, 11753-11759 (2008).

4. Oshima, M. \& Iwama, A. Epigenetics of hematopoietic stem cell aging and disease. Int J Hematol 100, 326-334 (2014).

5. Tsompana, M. \& Buck, M.J. Chromatin accessibility: a window into the genome. Epigenetics \& Chromatin 7, 33 (2014).

6. Voong, L.N., Xi, L., Wang, J.-P. \& Wang, X. Genome-wide Mapping of the Nucleosome Landscape by Micrococcal Nuclease and Chemical Mapping. Trends in Genetics 33, 495-507 (2017).

7. Ryu, Y.B. et al. Biflavonoids from Torreya nucifera displaying SARS-CoV 3CL(pro) inhibition. Bioorg Med Chem 18, 7940-7947 (2010).

8. Fejes, A.P. et al. FindPeaks 3.1: a tool for identifying areas of enrichment from massively parallel short-read sequencing technology. Bioinformatics 24, 17291730 (2008).

9. Wang, Z. et al. Combinatorial patterns of histone acetylations and methylations in the human genome. Nat Genet 40, 897-903 (2008).

10. Shipony, Z. et al. Long-range single-molecule mapping of chromatin accessibility in eukaryotes. Nat Methods 17, 319-327 (2020).

11. Lee, I. et al. Simultaneous profiling of chromatin accessibility and methylation on human cell lines with nanopore sequencing. bioRxiv, 504993 (2019).

12. D., K. et al. The Architecture of SARS-CoV-2 Transcriptome. Cell 181 (2020).

13. Shilatifard, A. Chromatin Modifications by Methylation and Ubiquitination: Implications in the Regulation of Gene Expression. Annual Review of Biochemistry 75, 243-269 (2006).

14. Mardis, E.R. ChIP-seq: welcome to the new frontier. Nat Methods 4, 613-614 (2007).

15. Johnson, D.S., Mortazavi, A., Myers, R.M. \& Wold, B. Genome-Wide Mapping of in Vivo Protein-DNA Interactions. Science 316, 1497 (2007).

16. Mikkelsen, T.S. et al. Genome-wide maps of chromatin state in pluripotent and lineage-committed cells. Nature 448, 553-560 (2007).

17. Barski, A. et al. High-resolution profiling of histone methylations in the human genome. Cell 129, 823-837 (2007).

18. Schones, D.E. et al. Dynamic regulation of nucleosome positioning in the human genome. Cell 132, 887-898 (2008).

19. Park, P.J. ChIP-seq: advantages and challenges of a maturing technology. Nat Rev Genet 10, 669-680 (2009). 
20. Robertson, G. et al. Genome-wide profiles of STAT1 DNA association using chromatin immunoprecipitation and massively parallel sequencing. Nat Methods 4, 651-657 (2007).

21. Kharchenko, P.V., Tolstorukov, M.Y. \& Park, P.J. Design and analysis of ChIP-seq experiments for DNA-binding proteins. Nat Biotechnol 26, 1351-1359 (2008).

22. Valouev, A. et al. Genome-wide analysis of transcription factor binding sites based on ChIP-Seq data. Nat Methods 5, 829-834 (2008).

23. Schmidl, C., Rendeiro, A.F., Sheffield, N.C. \& Bock, C. ChIPmentation: fast, robust, low-input ChIP-seq for histones and transcription factors. Nat Methods 12, 963965 (2015).

24. Skene, P.J. \& Henikoff, S. An efficient targeted nuclease strategy for highresolution mapping of DNA binding sites. Elife 6 (2017).

25. Kaya-Okur, H.S. et al. CUT\&Tag for efficient epigenomic profiling of small samples and single cells. Nat Commun 10, 1930 (2019).

26. Carter, B. et al. Mapping histone modifications in low cell number and single cells using antibody-guided chromatin tagmentation (ACT-seq). Nat Commun 10, 3747 (2019).

27. Harada, A. et al. A chromatin integration labelling method enables epigenomic profiling with lower input. Nat Cell Biol 21, 287-296 (2019).

28. $\mathrm{Ai}, \mathrm{S}$. et al. Profiling chromatin states using single-cell itChIP-seq. Nat Cell Biol 21, 1164-1172 (2019).

29. Statham, A.L. et al. Bisulfite sequencing of chromatin immunoprecipitated DNA (BisChIP-seq) directly informs methylation status of histone-modified DNA. Genome Res 22, 1120-1127 (2012).

30. Brinkman, A.B. et al. Sequential ChIP-bisulfite sequencing enables direct genomescale investigation of chromatin and DNA methylation cross-talk. Genome Res 22, 1128-1138 (2012).

31. Lhoumaud, P. et al. EpiMethylTag: simultaneous detection of ATAC-seq or ChIPseq signals with DNA methylation. Genome Biol 20, 248 (2019).

32. Murray, I.A. et al. The non-specific adenine DNA methyltransferase M.EcoGIl. Nucleic Acids Res 46, 840-848 (2018).

33. van Steensel, B. \& Henikoff, S. Identification of in vivo DNA targets of chromatin proteins using tethered dam methyltransferase. Nat Biotechnol 18, 424-428 (2000).

34. Douvlataniotis, K., Bensberg, M., Lentini, A., Gylemo, B. \& Nestor, C.E. No evidence for DNA N (6)-methyladenine in mammals. Sci Adv 6, eaay3335 (2020).

35. O'Brown, Z.K. et al. Sources of artifact in measurements of $6 \mathrm{~mA}$ and $4 \mathrm{mC}$ abundance in eukaryotic genomic DNA. BMC Genomics 20, 445 (2019).

36. Simpson, J.T. et al. Detecting DNA cytosine methylation using nanopore sequencing. Nat Methods 14, 407-410 (2017).

37. Rand, A.C. et al. Mapping DNA methylation with high-throughput nanopore sequencing. Nat Methods 14, 411-413 (2017).

38. Skene, P.J., Henikoff, J.G. \& Henikoff, S. Targeted in situ genome-wide profiling with high efficiency for low cell numbers. Nature Protocols 13, 1006-1019 (2018).

39. Kaya-Okur, H.S. et al. CUT\&Tag for efficient epigenomic profiling of small samples and single cells. Nature Communications 10, 1930 (2019). 
40. Furey, T.S. ChIP-seq and beyond: new and improved methodologies to detect and characterize protein-DNA interactions. Nature Reviews Genetics 13, 840-852 (2012).

41. Kouzarides, T. Chromatin modifications and their function. Cell 128, 693-705 (2007).

42. Berger, S.L. The complex language of chromatin regulation during transcription. Nature 447, 407-412 (2007).

43. Prickaerts, P. et al. Hypoxia increases genome-wide bivalent epigenetic marking by specific gain of H3K27me3. Epigenetics \& Chromatin 9, 46 (2016).

44. Young, M.D. et al. ChIP-seq analysis reveals distinct H3K27me3 profiles that correlate with transcriptional activity. Nucleic Acids Research 39, 7415-7427 (2011).

45. Holwerda, S.J. \& de Laat, W. CTCF: the protein, the binding partners, the binding sites and their chromatin loops. Philos Trans R Soc Lond B Biol Sci 368, 20120369 (2013).

46. Yin, M. et al. Molecular mechanism of directional CTCF recognition of a diverse range of genomic sites. Cell Res 27, 1365-1377 (2017).

47. Disdero, E. \& Filée, J. LoRTE: Detecting transposon-induced genomic variants using low coverage PacBio long read sequences. Mobile DNA 8, 5 (2017).

48. Pauler, F.M. et al. H3K27me3 forms BLOCs over silent genes and intergenic regions and specifies a histone banding pattern on a mouse autosomal chromosome. Genome Res 19, 221-233 (2009).

49. Román, A.C. et al. Dioxin receptor and SLUG transcription factors regulate the insulator activity of B1 SINE retrotransposons via an RNA polymerase switch. Genome Res 21, 422-432 (2011).

50. Kunarso, G. et al. Transposable elements have rewired the core regulatory network of human embryonic stem cells. Nature Genetics 42, 631-634 (2010).

51. Buenrostro, J.D., Wu, B., Chang, H.Y. \& Greenleaf, W.J. ATAC-seq: A Method for Assaying Chromatin Accessibility Genome-Wide. Curr Protoc Mol Biol 109, 21.29.21-21.29.29 (2015).

52. Klemm, S.L., Shipony, Z. \& Greenleaf, W.J. Chromatin accessibility and the regulatory epigenome. Nat Rev Genet 20, 207-220 (2019).

53. Vendrell, J.A. et al. ZNF217 is a marker of poor prognosis in breast cancer that drives epithelial-mesenchymal transition and invasion. Cancer Res 72, 3593-3606 (2012).

54. Krig, S.R. et al. ZNF217, a candidate breast cancer oncogene amplified at 20q13, regulates expression of the ErbB3 receptor tyrosine kinase in breast cancer cells. Oncogene 29, 5500-5510 (2010).

55. Voigt, P., Tee, W.W. \& Reinberg, D. A double take on bivalent promoters. Genes Dev 27, 1318-1338 (2013).

56. Dunican, D.S. et al. Bivalent promoter hypermethylation in cancer is linked to the H327me3/H3K4me3 ratio in embryonic stem cells. BMC Biol 18, 25 (2020).

57. Chapman-Rothe, N. et al. Chromatin H3K27me3/H3K4me3 histone marks define gene sets in high-grade serous ovarian cancer that distinguish malignant, tumoursustaining and chemo-resistant ovarian tumour cells. Oncogene 32, 4586-4592 (2013). 
58. Li, F. et al. Bivalent Histone Modifications and Development. Curr Stem Cell Res Ther 13, 83-90 (2018).

59. Adams, J. et al. Vascular endothelial growth factor (VEGF) in breast cancer: comparison of plasma, serum, and tissue VEGF and microvessel density and effects of tamoxifen. Cancer Res 60, 2898-2905 (2000).

60. Burger, J.A. \& Wiestner, A. Targeting B cell receptor signalling in cancer: preclinical and clinical advances. Nat Rev Cancer 18, 148-167 (2018).

61. Schütz, F. et al. PD-1/PD-L1 Pathway in Breast Cancer. Oncol Res Treat 40, 294297 (2017).

62. Hosogane, M., Funayama, R., Shirota, M. \& Nakayama, K. Lack of Transcription Triggers H3K27me3 Accumulation in the Gene Body. Cell Reports 16, 696-706 (2016).

63. Hall, I.M.J.B. BEDTools: a flexible suite of utilities for comparing genomic features. 26, 841 (2010).

64. Langmead, B. \& Salzberg, S.L.J.N.M. Fast gapped-read alignment with Bowtie 2. 9, 357-359 (2012).

65. Feng, J., Liu, T., Qin, B., Zhang, Y. \& Liu, X.S.J.N.P. Identifying ChIP-seq enrichment using MACS. 7, 1728 (2012).

66. Meers, M.P., Tenenbaum, D., Henikoff, S.J.E. \& Chromatin Peak calling by Sparse Enrichment Analysis for CUT\&RUN chromatin profiling. (2019).

67. Chen, X. et al. KOBAS 2.0: a web server for annotation and identification of enriched pathways and diseases. 39, 316-322 (2011).

68. Sedlazeck, F.J. et al. Accurate detection of complex structural variations using single-molecule sequencing. (2018).

69. Lei, B., Mi, Z. \& Cui, Y.J.N.A.R. CTCFBSDB: a CTCF-binding site database for characterization of vertebrate genomic insulators. 36, D83-87 (2008). 
bioRxiv preprint doi: https://doi.org/10.1101/2021.07.08.451578; this version posted July 15, 2021. The copyright holder for this preprint (which was not certified by peer review) is the author/funder, who has granted bioRxiv a license to display the preprint in perpetuity. It is made available under aCC-BY-NC-ND 4.0 International license.

A

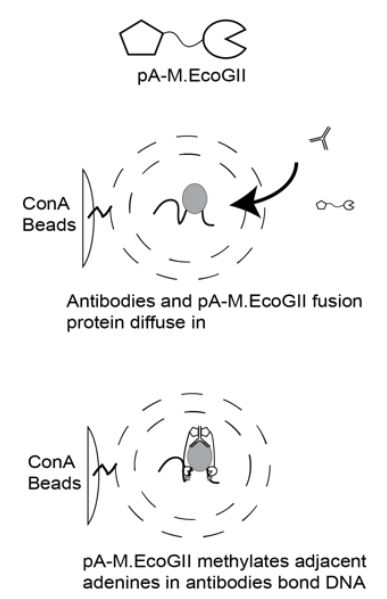

D

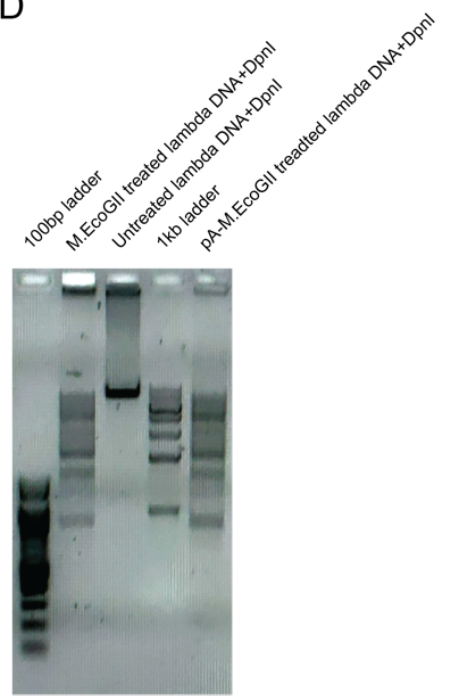

B

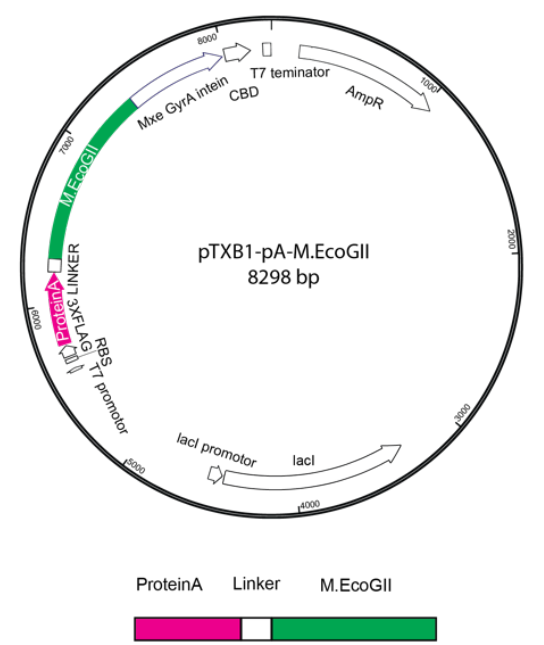

E

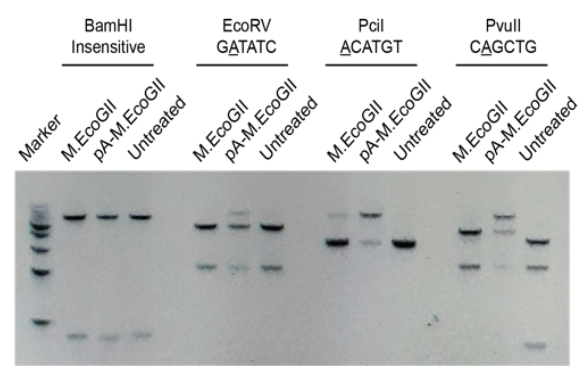

F

\begin{tabular}{|l|l|l|}
\hline Samples (OD450) & $\begin{array}{l}\text { Dilution } \\
1 / 120\end{array}$ & $\begin{array}{l}\text { Dilution } \\
\mathbf{1} / 480\end{array}$ \\
\hline pA-M.EcoGII & 2.238 & 1.939 \\
\hline M.EcoGII & 0.121 & 0.122 \\
\hline Negative control & 0.119 & 0.122 \\
\hline ProteinA & 0.799 & 0.305 \\
\hline
\end{tabular}

C

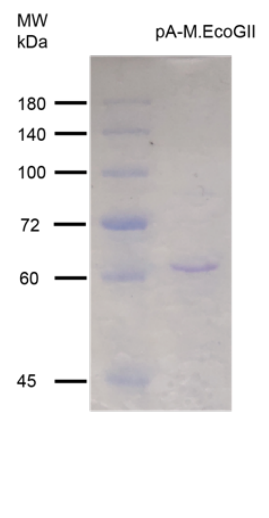

G

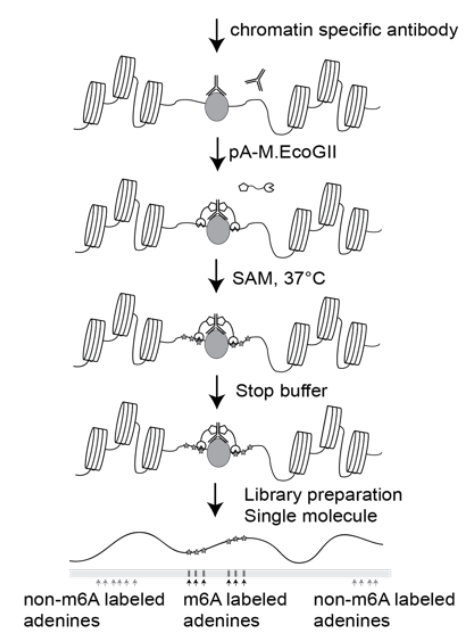


1 Figure 1. The experiment concept and validation of the recombinant protein pA-

2 M.EcoGII in BIND\&MODIFY. (A)The experiment concept of the BIND\&MODIFY. The

3 cells were lightly fixed and permeabilized for the antibody and enzyme passage. The

4 recombinant protein $\mathrm{pA}-\mathrm{M}$. EcoGII was located to desired sites under antibody guidance.

5 Then the M.EcoGIl activity was locally activated to modify the nearby regions to label

6 the genomic DNA with targeted binding proteins. (B) The upper panel showed the

7 plasmid map of the pA-M.EcoGII. The fused pA-M.EcoGII was cloned into pTXB1

8 plasmid and purified with compatible IMPACT protein purification system. The lower

9 panel showed the expressed fusion protein structure: Protein A-linker-M.EcoGIl-intein-

10 CBD. (C) The Coomassie blue gel stain showed the purity of the purified protein A-

11 M.EcoGII. (D) Methylation of linear lambda DNA by pA-M.EcoGIl activates m6A-site

12 dependent Dpnl restriction endonuclease digestion. The PCR amplified unmethylated

13 lambda DNA was treated with commercial M.EcoGII, no enzyme, and pA-M.EcoGII. The

14 GATC m6A methylation dependent restriction endonuclease Dpnl digestion showed the

15 comparable methyltransferase activity of the commercial M.EcoGII and our recombinant

16 proteins. DNA marker: 100bp ladder, 100-1510bp(left); 1kb ladder, 250-10,000bp(right).

17 (E) Methylation of linear dsDNA by pA-M.EcoGII inhibits multiple site-specific

18 methylation sensitive restriction endonucleases. The unmethylated DNA template was a

$197 \mathrm{~kb}$ linear dsDNA, which was PCR amplified from pTXB1 plasmid. The DNA template

20 was treated with commercial M.EcoGII, pA-M.EcoGIl and no enzyme. These treated

21 DNA templates were each incubated with four restriction endonucleases (BamHI,

22 EcoRV, Pcil, Pvull). The BamHI is the m6A methylation insensitive enzyme, and the

23 EcoRV, Pcil, Pvull are the m6A methylation sensitive enzyme, with which the digestion 
1 could be blocked by corresponding m6A site. Our pA-M.EcoGll recombinant protein

2 showed digestion inhibition on EcoRV, Pcil, Pvull digested samples, better than

3 commercial M.EcoGII, as compared to untreated DNA template. DNA marker, $1 \mathrm{~kb}$

4 ladder, 250-10,000bp. (F) The antibody affinity assay showed the recombinant pA-

5 M.EcoGll had the affinity to the secondary antibody in two different dilutions

$6(1 / 120,1 / 480,10 \mathrm{mg} / \mathrm{ml})$. (G) The experiment outlines of BIND\&MODIFY. After light

7 fixation and permeabilization, the cells were tethered to Concanavalin A magnetic

8 beads for the purification in the next steps. Then the cells were incubated with antibody

9 and pA-M.EcoGIl with minimal washes. The addition of S-adenosylmethionine to

10 initialize the methylation reaction. The DNA was extracted to prepare the library for ONT

11 nanopore sequencing. After sequencing, the data was processed as genome alignment

12 and m6A base calling. 
A

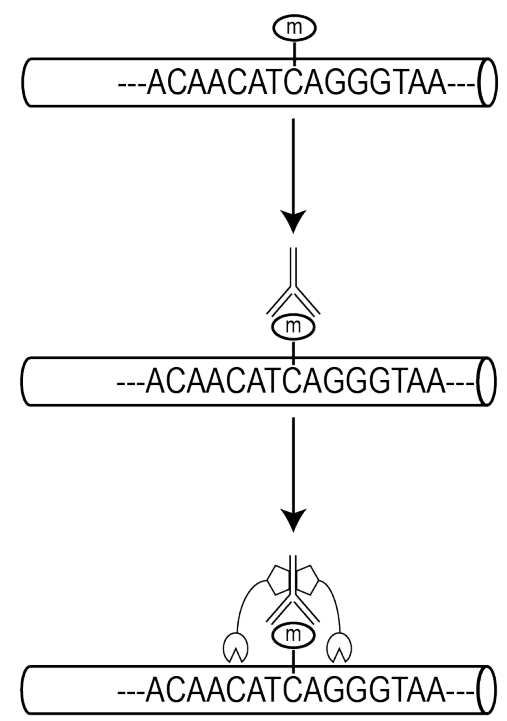

1

\section{2}
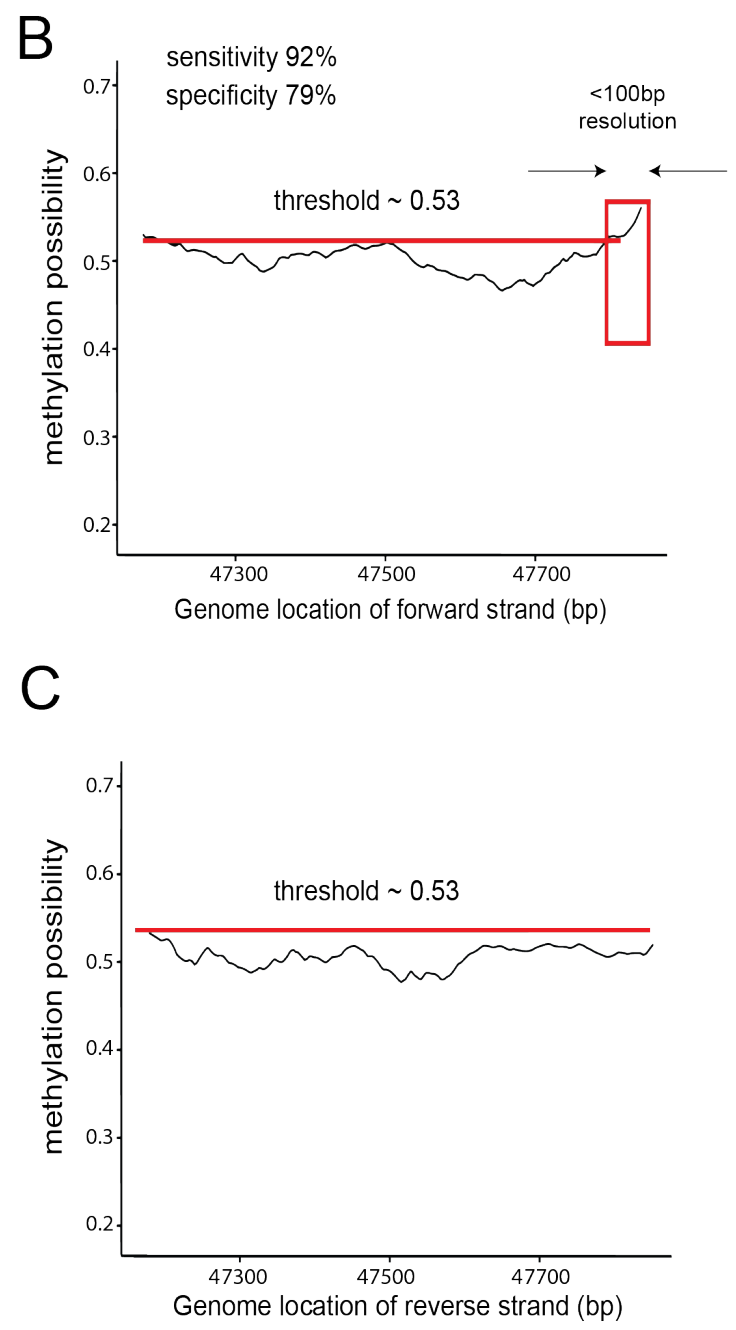

Figure 2. The experimental validation of the m6A calling in BIND\&MODIFY in vitro.

(A) Schematic outline of in vitro validation experiment. A fragment of $700 \mathrm{bp}$ lambda

DNA was amplified by PCR, and 5mC was introduced at 5' end of forward strand only.

The $5 \mathrm{mC}$ labeled DNA was bound by $5 \mathrm{mC}$ antibody and was subsequently treated by

BIND\&MODIFY method. (B) The $\mathrm{m}^{6} \mathrm{~A}$ possibility (Megalodon calling possibility) of

forward strand. When the methylation cut-off was set at 0.53 (red line, sensitivity=0.92,

specificity=0.79), high methylation possibility region was observed in 3' end at resolution

of about $100 \mathrm{bp}$, which was originally marked by $5 \mathrm{mC}$ at 3 ' end. (C) The methylation 
bioRxiv preprint doi: https://doi.org/10.1101/2021.07.08.451578; this version posted July 15, 2021. The copyright holder for this preprint (which

was not certified by peer review) is the author/funder, who has granted bioRxiv a license to display the preprint in perpetuity. It is made available under aCC-BY-NC-ND 4.0 International license.

1 possibility of reverse strand. No high methylation possibility region was observed above

20.53 cut-off.

3 
bioRxiv preprint doi: https://doi.org/10.1101/2021.07.08.451578; this version posted July 15, 2021. The copyright holder for this preprint (which was not certified by peer review) is the author/funder, who has granted bioRxiv a license to display the preprint in perpetuity. It is made available under aCC-BY-NC-ND 4.0 International license.

A

chr20_45,609,343-49,644,100_4028Kb

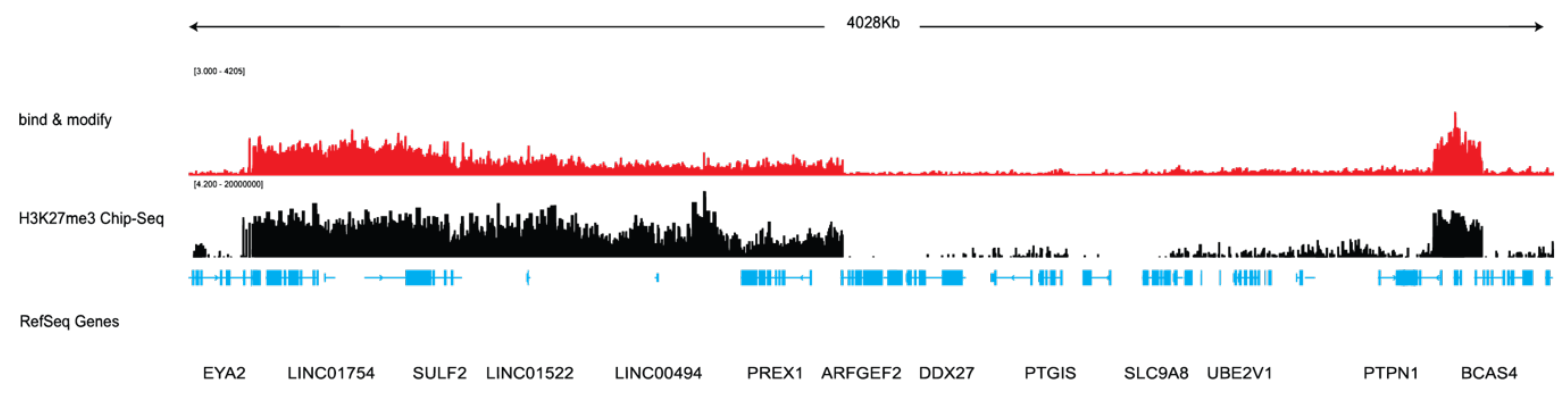

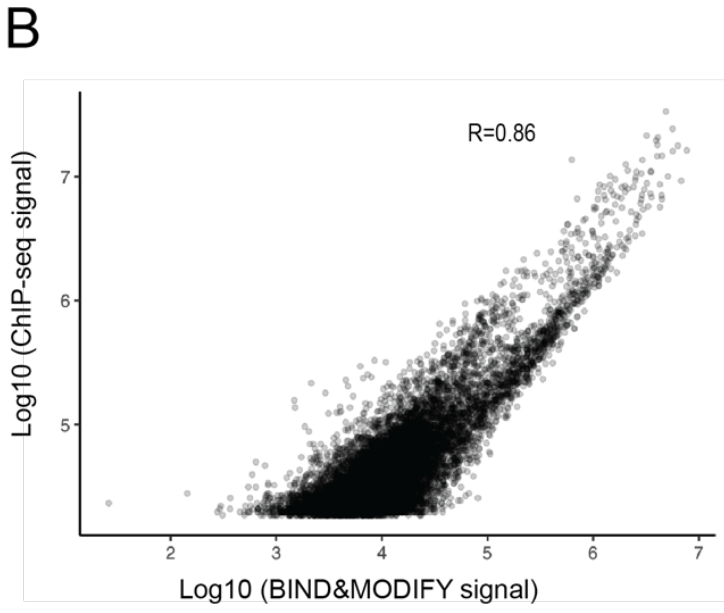

D

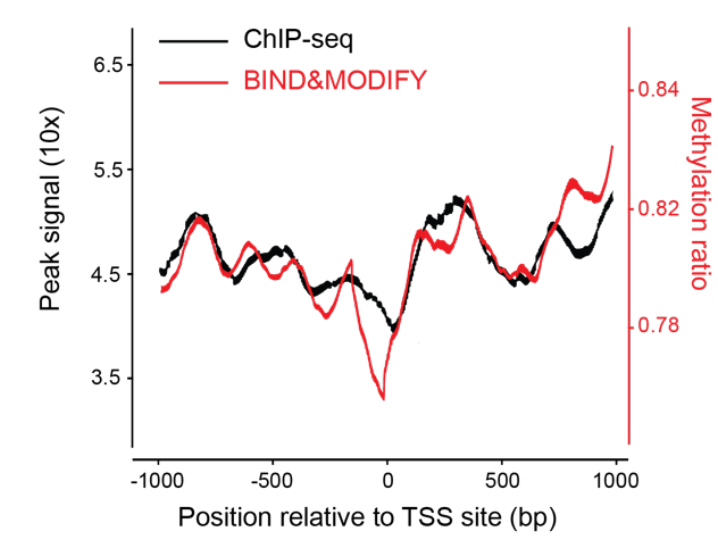

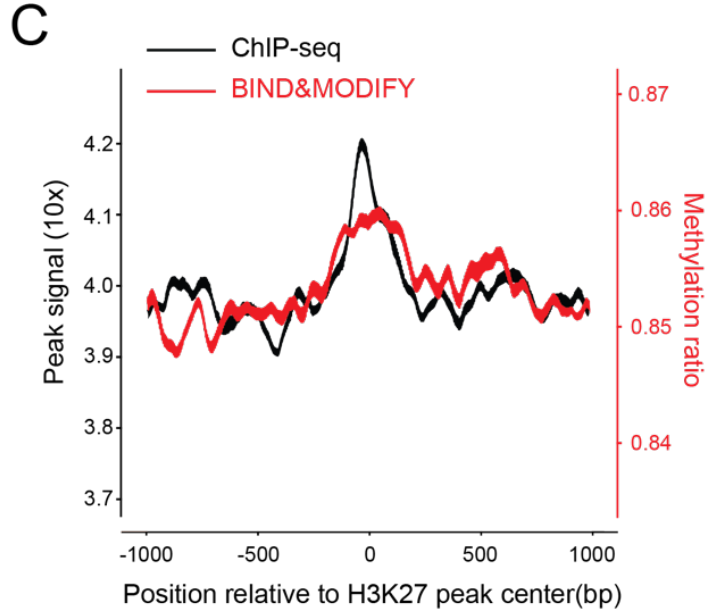

E

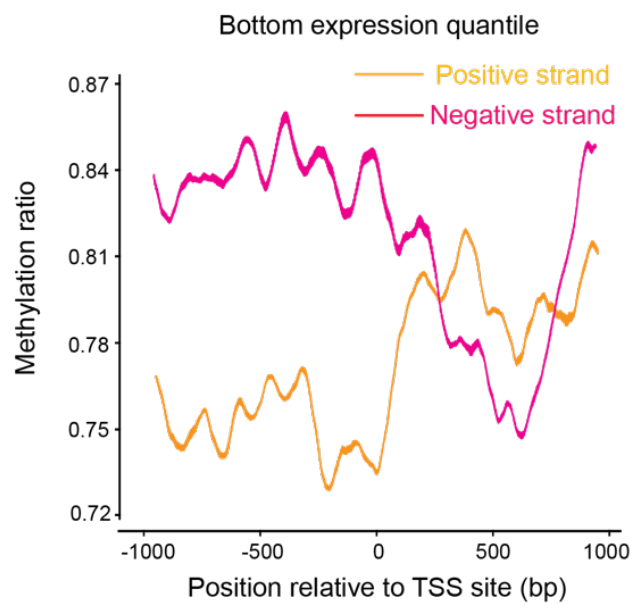


1 Figure 3. The consistency of H3K27me3 pattern between ChIP-seq and

2 BIND\&MODIFY in vivo. (A) The H3K27me3 signal, by BIND\&MODIFY and ChIP-seq,

3 in genome scale view. (B) The scatter plot of BIND\&MODIFY signal and ChIP-seq

4 signal in peak regions. The peak regions are identified H3K27me3 peaks in ChIP-seq.

5 The BIND\&MODIFY signal means the m6A counts and the ChIP-seq signal means the

6 read counts. (C) The H3K27me3 pattern of BIND\&MODIFY methylation ratio and ChIP-

7 seq signal was plotted by the H3K27me3 peak centered plot (H3K27me3 peaks

8 identified in ChIP-seq) for all the genes on Chr20. The plot covered the

9 upstream/downstream 1000bp from H3K27me3 peak center. (D) The H3K27me3

10 pattern of BIND\&MODIFY methylation ratio and ChIP-seq signal was plotted by the TSS

11 centered plot for the low expression genes. The BIND\&MODIFY line was the fitting

12 curve based on the positive strand and negative strand contribution on each region. The

13 low expression genes are the genes in the bottom expression quantile. (E) The

14 H3K27me3 strand-specific view by the TSS centered plot for the low expression genes. 
bioRxiv preprint doi: https://doi.org/10.1101/2021.07.08.451578; this version posted July 15, 2021. The copyright holder for this preprint (which was not certified by peer review) is the author/funder, who has granted bioRxiv a license to display the preprint in perpetuity. It is made available under aCC-BY-NC-ND 4.0 International license.

A

normalized peak signal resolution $\sim 0.5$

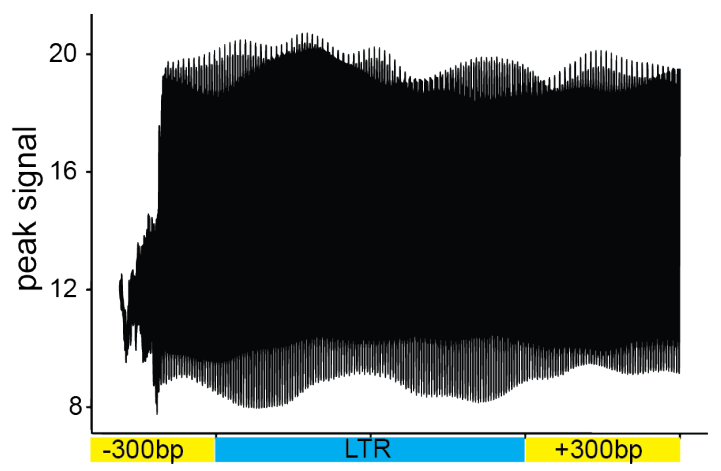

C

1

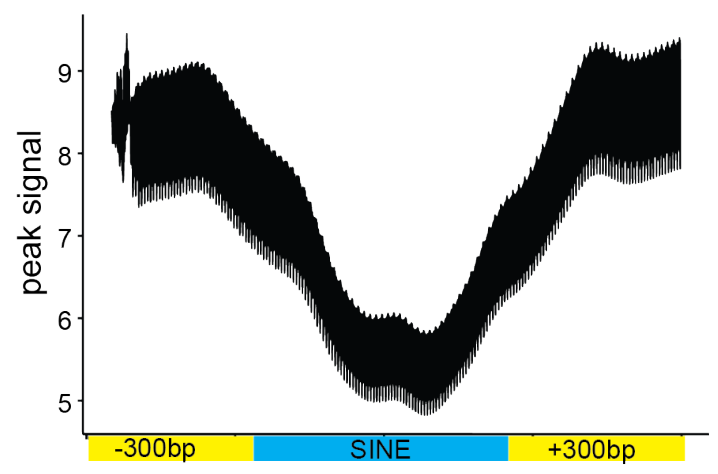

B normalized methylation ratio resolution $\sim 0.045$

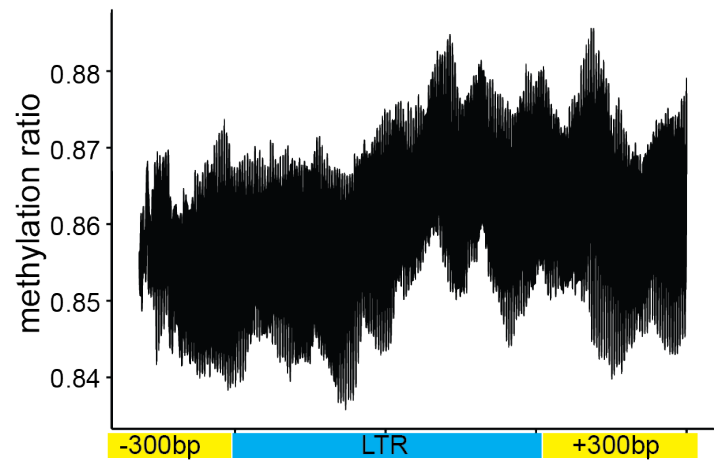

D

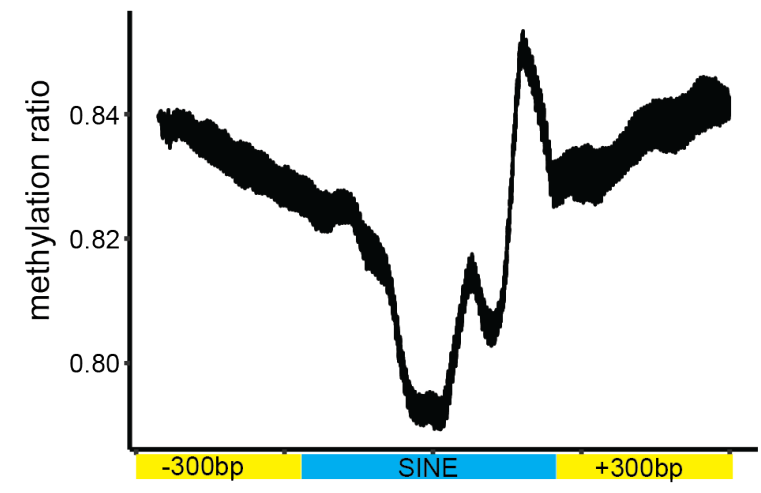


1 Figure 4. The BIND\&MODIFY resolved H3K27me3 in the transposon areas with

2 higher resolution. (A-B) The LTRs with size 350 450bp were selected and centered.

3 The moving average H3K27me3 signals on the upstream/downstream 300bp of these

4 LTRs, including LTRs, were plotted with corresponding genomic sites. The y-axis (A)

5 indicated the normalized read counts of ChIP-seq. The $y$-axis (B) indicated the

6 normalized methylation ratio of $\mathrm{m6A}$ in BIND\&MODIFY with nanopore sequencing.

7 Normalized peak signal resolution is defined by ratio of mean peak signal width

8 (maximum-minimum) and mean maximum peak signal, which is about 0.5 in ChIP-seq

9 and 0.045 in BIND\&MODIFY. (C-D) The SINEs with size 250 350bp were selected and

10 centered. The moving average H3K27me3 signal on the upstream/downstream 300bp

11 of these SINEs, including SINEs, were plotted with corresponding genomic sites. The y-

12 axis $(C)$ indicated the normalized read counts of short-reads ChIP-seq. The y-axis (D)

13 indicated the normalized methylation ratio of $\mathrm{m6A}$ in BIND\&MODIFY with nanopore

14 sequencing. 
bioRxiv preprint doi: https://doi.org/10.1101/2021.07.08.451578; this version posted July 15, 2021. The copyright holder for this preprint (which was not certified by peer review) is the author/funder, who has granted bioRxiv a license to display the preprint in perpetuity. It is made available under aCC-BY-NC-ND 4.0 International license.

A

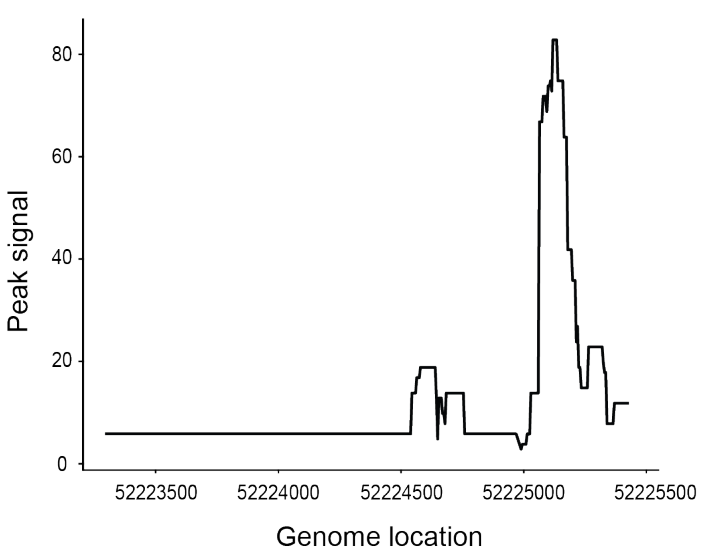

C
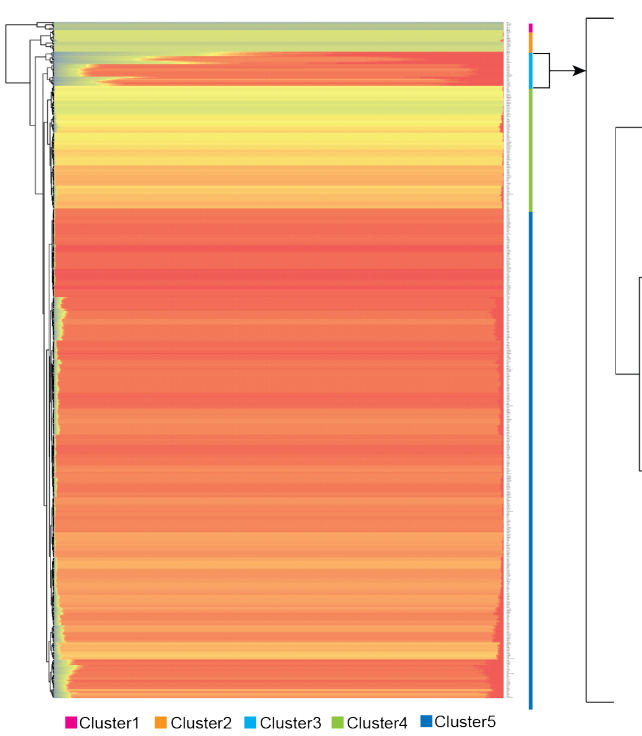

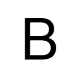

chr20:52,183,610-52,210,378 (GRCh37/hg19 by Entrez Gene)

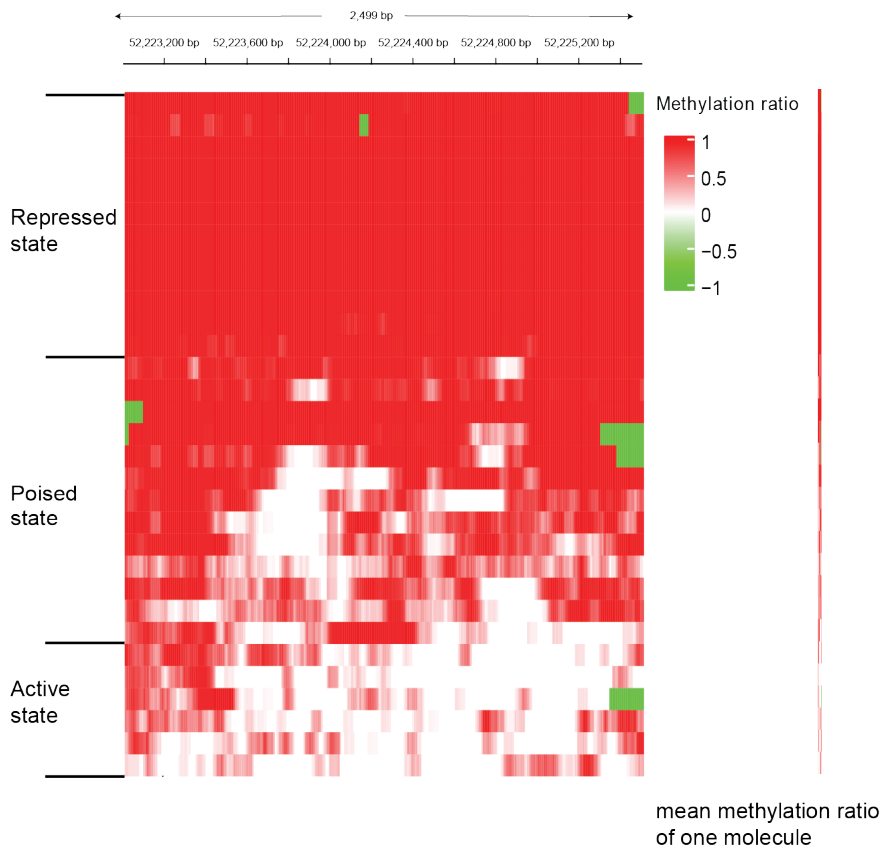

D

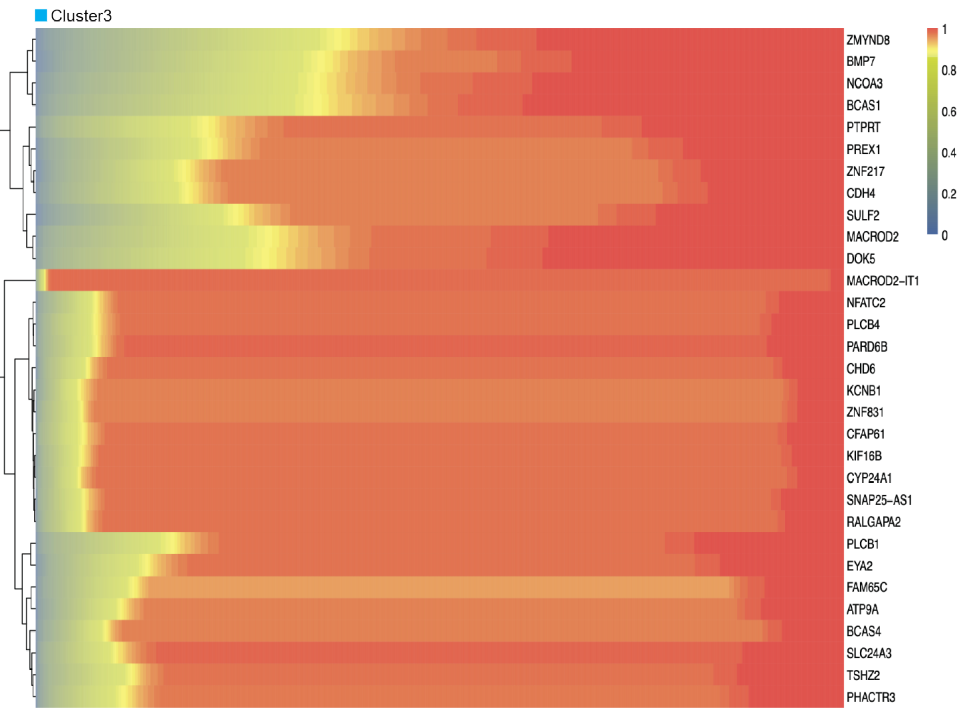


1 Figure 5. The BIND\&MODIFY showed the heterogeneity of $\mathrm{H} 3 \mathrm{~K} 27 \mathrm{me} 3$ regulation.

2 (A) The peak signal of H3K27me3 in ChIP-seq (Chr20:52,183,610-52,210,378). (B) The

3 single molecular resolution of the positive strand Chr20:52,183,610-52,210,378

4 visualize each molecular methylation statues of H3K27me3. The rows in heatmap

5 represented the different DNA molecules. The color indicated the methylation ratio,

6 which represented the H3K27me3 signal. The DNA molecules could be classified into

7 three states: repressed state, poised state, and active state based on their mean

8 methylation ratio. The right panel is the condensed illustration of individual DNA

9 molecules mean methylation ratio. Each block on the vertical line (molecule

10 heterogeneity line) represented the mean methylation of each DNA molecule. (C)

$11 \mathrm{H} 3 \mathrm{~K} 27$ me3 heterogeneity pattern of genes in chr20. Mean H3K27me3 methylation ratio

12 of individual DNA molecule was plotted for all the genes on DNA region

13 (Chr20:52,183,610-52,210,378). Each pixel on each row corresponds to mean

14 methylation ratio of each individual DNA molecule, and each row corresponds to each

15 gene. The methylation ratio was ranked from low to high (left to right). (D) The right

16 heatmap showed the magnified cluster 3 in the left heatmap. Details of H3K27me3

17 heterogeneity calculation methods can be found in supplementary figure 17 . 
bioRxiv preprint doi: https://doi.org/10.1101/2021.07.08.451578; this version posted July 15,2021 . The copyright holder for this preprint (which was not certified by peer review) is the author/funder, who has granted bioRxiv a license to display the preprint in perpetuity. It is made available under aCC-BY-NC-ND 4.0 International license.

A chr20_52278000-52280500. (positive strand)

B
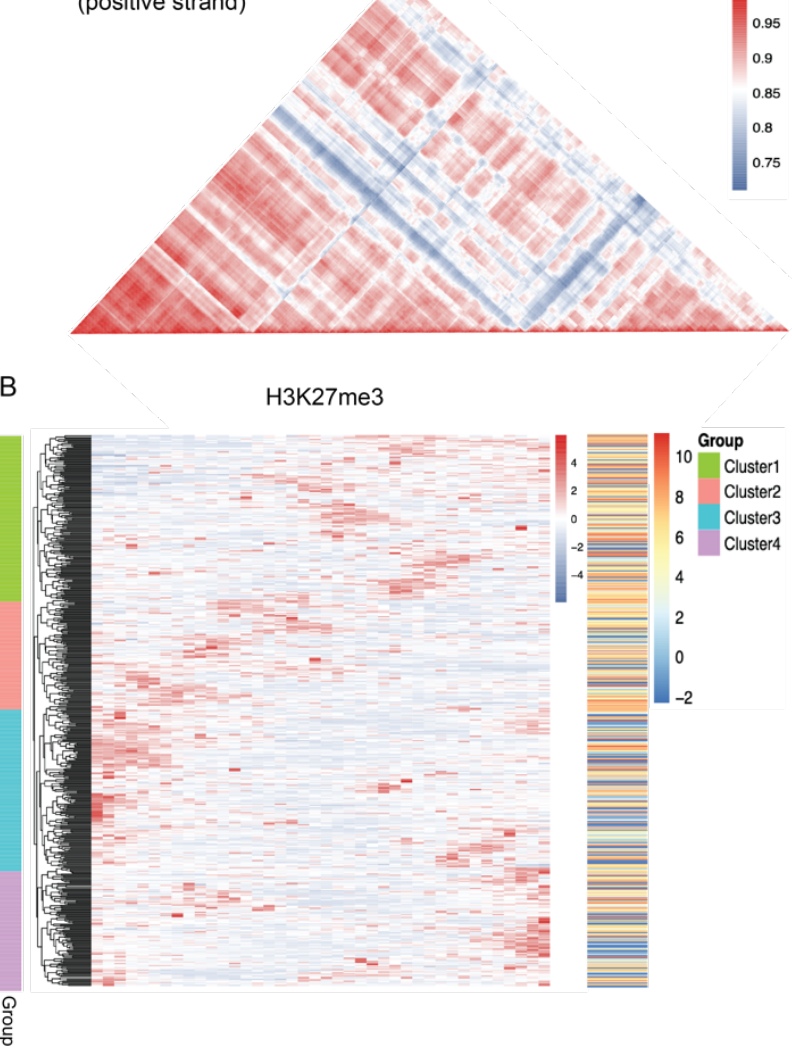

C

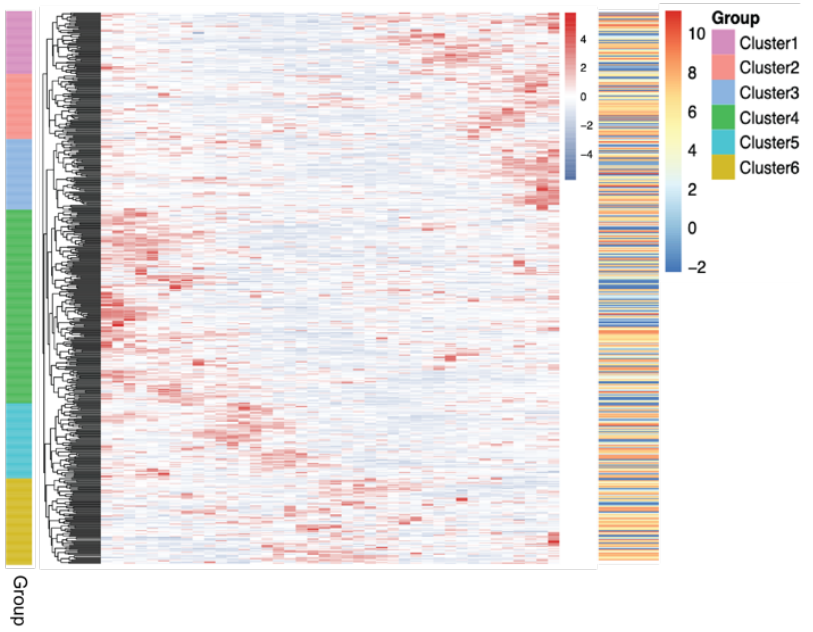


1 Figure 6. The distance correlation of the epigenetic regulation. (A) The distance

2 correlation of the H3K27me3 in chr20: 52278000 52280500 (positive strand). The color

3 indicated the correlation coefficient (CC) metric among genome regions. The higher CC

4 metric means the stronger correlation in distance. (B) The global view of the H3K27me3

5 distance effect (DE) index for all the genetic transcription regions (upstream $2 \mathrm{~kb}$ of

6 transcription start site) in chr20. The DE index is sum of the $C^{*}$ distance, representing

7 this location impact on other sites. The red color means the stronger impact on distal

8 genomic sites. The left color bar indicated the clusters of these transcription regions.

9 The right color bar indicated the corresponding gene expression. (C) The global view of

10 the CTCF distance effect for all the genetic transcription in chr20. Details of correlation

11 coefficient (CC) metric and distance effect (DE) index calculation method can be found

12 in supplementary figure 21. 